\title{
ON UNIFORM APPROXIMATION OF HARMONIC FUNCTIONS
}

\author{
M. YA. MAZALOV
}

\begin{abstract}
The paper is devoted to uniform approximation by harmonic functions on compact sets. The result is an approximation theorem for an individual function under the condition that, on the complement to the compact set, the harmonic capacity is "homogeneous" in a sense. The proof involves a refinement of Vitushkin's localization method.
\end{abstract}

\section{$\S 0$. INTRODUCTION}

Let $X \subset \mathbb{R}^{3}$ be a compact set, $X^{\circ}$ the interior of $X, \Delta$ the Laplace operator in $\mathbb{R}^{3}$. We define two classes of functions $h(X)$ and $H(X)$ as follows:

$$
h(X)=C(X) \cap\left\{\left.f\right|_{X}: \Delta f=0 \text { in } X^{\circ}\right\},
$$

and $H(X)$ is the closure in $C(X)$ of the set

$$
\left\{\left.f\right|_{X}: \Delta f=0 \text { in a neighborhood of } X\right\}
$$

(the neighborhood depends on $f$ ).

Clearly, $H(X) \subset h(X)$. There is a criterion for the identity $H(X)=h(X)$, namely, the Keldysh-Deny capacity criterion (see [1, 2]), which is similar to Vitushkin's criterion for analytic functions; see [3, Chapter 5, §3, Theorem 1]. Specifically,

$$
H(X)=h(X) \Longleftrightarrow \operatorname{Cap}\left(D \backslash X^{\circ}\right)=\operatorname{Cap}(D \backslash X),
$$

where $\operatorname{Cap}(\cdot)$ is harmonic capacity (see (1.3) ) and $D$ is an arbitrary bounded open set.

Consider the (more general) problem of description of all functions $f$ that belong to $H(X)$. The conjecture to be stated below was formulated, e.g., by O'Farrell [4]. Similar statements were established by Vitushkin (see [3, Chapter 4, §2, Lemma 1]) for uniform analytic approximation of individual functions, and by Paramonov [5, Theorem 5.1] for harmonic approximation in the $C^{1}$-norm.

There is no loss of generality in assuming that the function $f$ in question is extended to the entire $\mathbb{R}^{3}$ from $X$ up to a continuous compactly supported function (this can be done, e.g., by the Whitney theorem; see [6. Chapter 6, item 2.2]). Let $\omega_{f}$ be the modulus of continuity of the extended function $f$.

Conjecture 1. The condition $f \in H(X)$ is equivalent to the existence of positive constants $k$ and $k_{1}$ (which depend only on $f$ and $X$ ) with

$$
\left|\int_{B} f(x) \Delta \varphi(x) d m_{x}\right| \leq k_{1} \omega_{f}(r)\left\|\nabla^{2} \varphi\right\|_{\mathrm{L}_{\infty}} r^{2} \operatorname{Cap}(k B \backslash X),
$$

where $B$ is an arbitrary open ball in $\mathbb{R}^{3}$ of radius $r, x=\left(x_{1}, x_{2}, x_{3}\right) \in \mathbb{R}^{3}, m_{x}$ is the Lebesgue measure on $\mathbb{R}^{3}, \varphi$ is an arbitrary function in $C_{0}^{\infty}(B)$, and $k B$ is the ball homothetic to $B$ and of radius $\mathrm{kr}$.

2010 Mathematics Subject Classification. Primary 31B15, 31B05; Secondary 41A63.

Key words and phrases. Uniform approximation, harmonic functions, capacities, singular integrals, Carleson measures.

Supported in part by the grant NSh-3476.2010.1 for support of leading scientific schools. 
Since capacity characterizes the "size" of the set of irremovable singularities for harmonic functions (see, e.g., 7, Theorems 1.4 and 3.1]), we see that condition (0.1) can be interpreted in the following natural way: uniform approximation is possible if and only if the complement to the compact set is locally at least as "massive" as the set of singularities of the function.

The necessity of condition (0.1) for $f \in H(X)$ is proved in a standard way (see Lemma 1.3); moreover, we can put $k=1$, and $k_{1}$ is a universal constant. The arguments are similar to those in [3, Chapter 4, §2] or in [5, §5]. The question about sufficiency of (0.1) is much subtler than similar questions for uniform analytic approximation and for harmonic approximation in the $C^{1}$-norm (see Remark 1.3 in $\S 1$ ).

In the present paper, we verify a somewhat weaker version of Conjecture 1 .

Theorem 1. Suppose that condition (0.1) is fulfilled; moreover, suppose that there is a constant $k_{0} \geq 1$ (which may depend on $X$ ) such that for every $x \in \mathbb{R}^{3} \backslash X^{\circ}$ and all $r>0$ we have

$$
\operatorname{Cap}(B(x, 2 r) \backslash X) \leq k_{0} \operatorname{Cap}(B(x, r) \backslash X) .
$$

Then $f \in H(X)$.

It should be noted that, taken alone, estimate (0.2) does not imply that $f \in H(X)$ (see Example 1.1 in §1).

Theorem[1]will be proved with help of Vitushkin's method [3], which consists of decomposing the function in question into a sum of localizations and subsequently estimating and equating Laurent coefficients of these localizations. It should be noted that a substantial refinement of the method was required. In the present paper, we shall use a theorem on approximation of a function "by parts" [8, Theorem 2], which "improves the order by 1 " in Lemma 1 in [3, Chapter 2, §4]. However, the most important technical tool is the use of Calderón-Zygmund singular integrals on a specially constructed Lipschitz surface (see $\S 2$ ) for estimating and equating Laurent coefficients.

Note also that the geometric construction here is much more complicated than that in the papers [8, 9, where related but simpler statements were proved (for example, in [9], Verdera's conjecture (see [10]) was verified). In those papers differential operators with locally bounded fundamental solutions were considered (such as powers of the CauchyRiemann operator), and capacity conditions were not required.

We remark that there is no need to use all functions $\varphi$ occurring in (0.1) in the proof of Theorem 1: these functions arise naturally when we construct partitions of unity, their derivatives may be assumed to obey "fairly regular" estimates (see Lemma 1.1), and their number is finite for given $f$ and $X$. In fact, by analogy with [11, Theorem 2.2], we may restrict ourselves to functions $\varphi$ generated by one "test function of simple structure" via translations and shifts (see $\S 4$ ). The selection of a due test function leads to the following condition (0.3), which is more natural than (0.1) in applications.

Theorem 2. Suppose that condition (0.2) is satisfied and, in the notation of (0.1), the following is true:

$$
\left|\frac{1}{\sigma(\partial B)} \int_{\partial B} f(x) d \sigma_{x}-\frac{1}{m(B)} \int_{B} f(x) d m_{x}\right| \leq k_{1} \omega_{f}(r) r^{-1} \operatorname{Cap}(k B \backslash X),
$$

where $\sigma_{x}$ is the surface measure on the boundary $\partial B$ of the ball B. Then $f \in H(X)$.

Condition (0.3) has been suggested by Paramonov, by analogy with condition (iv) in [12, Theorem 1.1].

Finally, it should be noted that the proof of Theorems 1 and 2 can be carried over to harmonic functions in $\mathbb{R}^{d}(d>3)$ with minor changes.

The author is deeply thankful to the referee for valuable remarks. 


\section{§1. Preparatory Results}

Recall that the fundamental solution for the Laplacian in $\mathbb{R}^{3}$ looks like this:

$$
E(x)=-\frac{1}{4 \pi|x|} .
$$

Let $g$ be a distribution such that $\operatorname{Spt}(\Delta g)$ is compact $(\operatorname{Spt}(\cdot)$ stands for the closure of the support of "."). Outside $\operatorname{Spt}(\Delta g), g$ is a (classical) harmonic function. If $\lim _{x \rightarrow \infty} g(x)=0$, then

$$
g=E *(\Delta g)
$$

this relation is understood in the distributional sense. (Indeed, $E$ being the fundamental solution, the distribution $g-E *(\Delta g)$ coincides with a function harmonic on $\mathbb{R}^{3}$ and vanishing at infinity. By the Liouville theorem, this function is identically zero.)

As in [7. Definition 1.1], we define harmonic capacity. Let $K \subset \mathbb{R}^{3}$ be a compact set. The quantity

$$
\operatorname{Cap}(K) \stackrel{\text { def }}{=}(4 \pi)^{-1} \sup _{g}\left\{|\langle\Delta g \mid 1\rangle|:\|g\|_{\mathrm{L}_{\infty}} \leq 1, \lim _{x \rightarrow \infty} g(x)=0, \operatorname{Spt}(\Delta g) \subset K\right\}
$$

is called the capacity of $K$. The angular brackets in (1.3) indicate the action of a compactly supported distribution on an infinitely differentiable function. Specifically,

$$
\langle\Delta g \mid 1\rangle=\int g(x) \Delta \varphi(x) d m_{x}
$$

where $\varphi$ is an arbitrary function in $C_{0}^{\infty}\left(\mathbb{R}^{3}\right)$ with $\varphi(x) \equiv 1$ in a neighborhood of $\operatorname{Spt}(\Delta g)$.

We recall the usual definition of capacity in potential theory:

$$
\operatorname{Cap}(K) \stackrel{\text { def }}{=} \sup _{\mu}\left\{\|\mu\|:\left\|\mu * \frac{1}{|x|}\right\|_{\mathrm{L}_{\infty}} \leq 1\right\},
$$

where $\|\mu\|$ is the total mass of a nonnegative Radon measure $\mu$ supported on $K$. Formally, Definition (1.4) is a partial case of (1.3) (see (1.2)).

However, it is known that Definitions (1.3) and (1.4) are equivalent (see, e.g., 7, §3]).

Note also that if we add the requirement $\mu * \frac{1}{|x|} \in C\left(\mathbb{R}^{3}\right)$ in (1.4), we still obtain a definition equivalent to (1.4) (see, for instance, [1, Chapter 5, Lemma 12] or [13, Chapter 3, Theorem 2 and Lemma 6]).

For a bounded set $U$, we put

$$
\operatorname{Cap}(U) \stackrel{\text { def }}{=} \sup _{K \subset U} \operatorname{Cap}(K)
$$

where $K$ stands for a compact set.

In this paper, we shall use well-known elementary properties of the capacity $\operatorname{Cap}(\cdot)$ (see, e.g., [1, Chapter 2, item 1, Properties 1-5]).

We remind the reader that the Vitushkin method consists of the following:

1 ) to split the function $f$ into a sum of localizations by using a partition of unity;

2) to approximate the localizations in a way ensuring the relation $f \in H(X)$.

By a partition of unity on a compact set $K$, we mean a finite collection of nonnegative functions $\varphi_{j} \in C_{0}^{\infty}\left(\mathbb{R}^{3}\right)$ such that $\sum_{j} \varphi_{j}(x) \equiv 1$ in a neighborhood of $K$.

Let $\left\{\varphi_{j}\right\}$ be a partition of unity on $\operatorname{Spt}(\Delta f)$. By (1.2), $f$ is represented as a sum of localizations

$$
f=\sum_{j} f_{j}, \quad \text { where } \quad f_{j}=E *\left(\varphi_{j} \Delta f\right)
$$


the operator $V_{\varphi}$,

$$
V_{\varphi} \Psi=E *(\varphi \Delta \Psi)
$$

where $\varphi \in C_{0}^{\infty}\left(\mathbb{R}^{3}\right), \Psi \in\left(C_{0}^{\infty}\left(\mathbb{R}^{3}\right)\right)^{\prime}$, is called a localization operator.

In this paper, we shall use partitions of unity invented by Harvey and Polking; see [14, Lemma 3.1] and also [15, $\S 2$, item $1^{\circ}$. (The required partition of unity depending on $X$ and $f$ will be introduced in $\S \S 2-3$.) As a preliminary, we make some comments.

In what follows, $\alpha=\left(\alpha_{1}, \alpha_{2}, \alpha_{3}\right)$ is a multiindex $\left(\alpha_{m} \in \mathbb{Z}_{+}, m=1,2,3\right)$,

$$
|\alpha|=\sum_{m=1}^{3} \alpha_{m}, \quad \partial^{\alpha}=\frac{\partial^{|\alpha|}}{\partial x_{1}^{\alpha_{1}} \partial x_{2}^{\alpha_{2}} \partial x_{3}^{\alpha_{3}}}, \quad \alpha !=\alpha_{1} ! \alpha_{2} ! \alpha_{3} !, \quad x^{\alpha}=x_{1}^{\alpha_{1}} x_{2}^{\alpha_{2}} x_{3}^{\alpha_{3}} .
$$

Throughout, by a cube we mean a closed cube with edges parallel to coordinate axes. For a cube $Q=Q(a, s)$ centered at $a$ and with side length $s$, we denote by $\lambda Q$ the cube with the same center and with side length $\lambda s$. The name dyadic cubes is reserved for the cubes of the form

$$
Q=Q_{p}^{m_{1}, m_{2}, m_{3}}=\prod_{k=1}^{3}\left[m_{k} 2^{-p},\left(m_{k}+1\right) 2^{-p}\right],
$$

where $\left(p, m_{1}, m_{2}, m_{3}\right) \in \mathbb{Z}^{4}$.

When we consider coverings of compact sets by finite families of dyadic cubes, we always assume that the cubes are disjoint, i.e., have mutually nonintersecting interiors.

Lemma 1.1 (see [14, Lemma 3.1]). Let $\left\{Q_{j}\right\}$ be a finite family of disjoint dyadic cubes. Then there exists a partition of unity $\left\{\varphi_{j}\right\}$ for the set $\bigcup_{j} Q_{j}$ such that $\operatorname{Spt} \varphi_{j} \subset(3 / 2) Q_{j}$ and $\left\|\nabla^{n} \varphi_{j}\right\|_{\mathrm{L}_{\infty}} \leq A(n)\left(s\left(Q_{j}\right)\right)^{-n}$ for all $n \in \mathbb{Z}_{+}$.

In what follows, $A, A_{0}, A_{1}, \ldots$ stand for positive universal constants. Their particular values may vary from one formula to another. The constants $k_{j}>0(j=0,1,2, \ldots)$ are fixed.

The next statement about properties of localizations is similar to Lemma 1 in 3 , Chapter 2, §3] and is proved in a standard way; see [5, §4], [15, Lemma 14.10], and [16. Proposition 2.5].

Lemma 1.2. Let $B=B(a, \delta)$ be a ball in $\mathbb{R}^{3}$, let $\varphi \in C_{0}^{\infty}(B)$, and let $g \in C\left(\mathbb{R}^{3}\right)$. Then the function $V_{\varphi} g$ (see (1.6) ) has the following properties:

a) $V_{\varphi} g \in C\left(\mathbb{R}^{3}\right)$ and $\lim _{x \rightarrow \infty} V_{\varphi} g(x)=0$;

b) $\Delta\left(V_{\varphi} g\right)=\varphi \Delta g$, and consequently, $\operatorname{Spt}\left(\Delta\left(V_{\varphi} g\right)\right) \subset(\operatorname{Spt} \varphi \cap \operatorname{Spt}(\Delta g))$;

c) we have

$$
\left\|V_{\varphi} g\right\|_{\mathrm{L}_{\infty}} \leq A \omega_{g}(\delta)\left\|\nabla^{2} \varphi\right\|_{\mathrm{L}_{\infty}} \delta^{2} .
$$

To estimate localizations outside the ball $B$, we use a Laurent series expansion in derivatives of the fundamental solution. We recall the principal facts; see [5, Lemma 3.2].

Let $T$ be a distribution supported on a ball $B(a, \delta)$, and let $h=T * E$. Then $h(x)$ admits an expansion in a Laurent series that converges in $C^{\infty}$ outside the ball $B(a, 3 \sqrt{3} \delta)$ :

$$
h(x)=\sum_{|\alpha| \geq 0} c_{\alpha} \partial^{\alpha} E(x-a),
$$

where the Laurent coefficients $c_{\alpha}$ are defined by

$$
c_{\alpha}=c_{\alpha}(h, a)=\frac{(-1)^{|\alpha|}}{\alpha !}\left\langle T(y) \mid(y-a)^{\alpha}\right\rangle .
$$


Formula (1.8) implies that, whenever $h \not \equiv 0$, there exists the maximal index $n$ such that

$$
h(x)=\sum_{|\alpha|=n} c_{\alpha} \partial^{\alpha} E(x-a)+o\left(|x|^{-1-n}\right)
$$

as $x \rightarrow \infty$. The coefficients $c_{\alpha}$ with $|\alpha|=n$ are called the senior Laurent coefficients of $h$; clearly, they do not depend on the center $a$ of expansion (the coefficient $c_{0}(h)=\langle T \mid 1\rangle$ never depends on the center of expansion).

Remark 1.1. By (1.2), an arbitrary function (1.8) such that $\operatorname{Spt}(\Delta h)$ is compact and $\lim _{x \rightarrow \infty} h(x)=0$ possesses a Laurent expansion (1.8).

In particular, for the function $V_{\varphi} g$ in Lemma 1.2 we have

$$
c_{\alpha}\left(V_{\varphi} g, a\right)=\frac{(-1)^{|\alpha|}}{\alpha !}\left\langle\varphi(y) \Delta g(y) \mid(y-a)^{\alpha}\right\rangle
$$

(moreover, $\left.c_{0}\left(V_{\varphi} g\right)=\langle\varphi \Delta g \mid 1\rangle\right)$.

From Lemma 1.2 and the definition of the capacity, we readily deduce the estimate

$$
\left|c_{0}\left(V_{\varphi} g\right)\right| \leq A \omega_{g}(\delta)\left\|\nabla^{2} \varphi\right\|_{\mathrm{L}_{\infty}} \delta^{2} \operatorname{Cap}(\operatorname{Spt} \varphi \cap \operatorname{Spt}(\Delta g)),
$$

and also the necessity of condition (0.1) for $f \in H(X)$.

Lemma 1.3. If $f \in H(X)$, then (0.1) is fulfilled with $k=1$ and a universal constant in the role of $k_{1}$.

Proof. Let $f \in H(X)$. Then for every $\varepsilon>0$ there is a function $F$ such that $\Delta F=0$ in a neighborhood of $X$ and $|f(x)-F(x)|<\varepsilon$. Reducing $\varepsilon$ if necessary and extending $f-F$ by the Whitney theorem [6, Chapter 6, item 2.2] (like we did with $f$ ), we may assume that everywhere on $\mathbb{R}^{3}$ we have $|f(x)-F(x)| \leq \omega_{f}(\delta)$.

Estimating the functions $V_{\varphi} f=E *(\varphi \Delta f)$ and $E *(\varphi \Delta(f-F))$ by Lemma 1.2, we obtain a due estimate for $V_{\varphi} F$ :

$$
\left\|V_{\varphi} F\right\|_{\mathrm{L}_{\infty}} \leq A \omega_{f}(\delta)\left\|\nabla^{2} \varphi\right\|_{\mathrm{L}_{\infty}} \delta^{2} .
$$

Since $\Delta\left(V_{\varphi} F\right)=\varphi \Delta F$ (consequently, $\left.\operatorname{Spt}\left(\Delta\left(V_{\varphi} F\right)\right) \subset(B \backslash X)\right)$, by (1.10) and (1.11) we obtain

$$
\left|\int_{B} F(x) \Delta \varphi(x) d m_{x}\right|=|\langle\varphi \Delta F \mid 1\rangle|=\left|c_{0}\left(V_{\varphi} F\right)\right| \leq A \omega_{f}(\delta)\left\|\nabla^{2} \varphi\right\|_{\mathrm{L}_{\infty}} \delta^{2} \operatorname{Cap}(B \backslash X) .
$$

Since $|f(x)-F(x)|<\varepsilon$ and $\varepsilon$ is arbitrary, this yields a similar estimate for the left-hand side of (0.1).

Condition (0.1) is convenient for the verification of the property that a function in $h(K)$ does not belong to $H(K)$.

Example 1.1. There exists a compact set $K \subset \mathbb{R}^{3}$ and a function $f \in h(K)$ such that (0.2) is fulfilled for $K$ but $f \notin H(K)$.

Construction of the example. We start with elementary estimates. Let $C$ be a right circular cylinder with base of radius $r$ and of height $a, a \geq 2 r$. Then (see, e.g., [17, Chapter 2, §3, item 14]) there is a constant $A>1$ such that

$$
A^{-1} \frac{a}{\log \frac{a}{r}} \leq \operatorname{Cap}\left(C^{\circ}\right) \leq A \frac{a}{\log \frac{a}{r}} .
$$

In particular, if $a \leq 1$ and $a / r=\exp \left(1 / a^{2}\right)$, we have $A^{-1} a^{3} \leq \operatorname{Cap}\left(C^{\circ}\right) \leq A a^{3}$. 
For $t \in(0,1 / 10]$, let $U\left(t_{1}, t_{2}, t\right)$ be the open set bounded "from below" by the surface of revolution $x_{3}=\sqrt{\left(x_{1}-t_{1}\right)^{2}+\left(x_{2}-t_{2}\right)^{2}} \exp \left(1 /\left(x_{3}\right)^{2}\right)$, and "from above" by the plane $x_{3}=t$; the above estimate for the capacity of a cylinder shows that

$$
\left(A_{1}\right)^{-1} t^{3} \leq \operatorname{Cap}\left(U\left(t_{1}, t_{2}, t\right)\right) \leq A_{1} t^{3}
$$

with a universal constant $A_{1}>1$.

We take

$$
K=\overline{B(0,10)} \backslash \bigcup_{m_{1}, m_{2}, n} U\left(\frac{m_{1}}{10^{n}}, \frac{m_{2}}{10^{n}}, \frac{1}{10^{n}}\right),
$$

where $\left(m_{1}, m_{2}, n\right) \in \mathbb{N}^{3}$ and $m_{1} / 10^{n}, m_{2} / 10^{n}$ are proper irreducible fractions. Let $\widetilde{Q}$ be the unit square of the plane $O x_{1} x_{2}$. Clearly, $\widetilde{Q}$ is included in the boundary of $K$, and the set $\widetilde{Q} \backslash \bigcup_{m_{1}, m_{2}, n}\left(\frac{m_{1}}{10^{n}}, \frac{m_{2}}{10^{n}}\right)$ is the internal boundary of $K$.

By (1.12), for every fixed $n$ and for an arbitrary disk of radius $r \geq 10^{-n+1}$ lying on the plane $O x_{1} x_{2}$ and centered at a point of $\widetilde{Q}$, we have

$$
\left(A_{2}\right)^{-1} r^{2} 10^{-n} \leq \sum_{m_{1}, m_{2}} \operatorname{Cap}\left(U\left(m_{1} / 10^{n}, m_{2} / 10^{n}, 1 / 10^{n}\right)\right) \leq A_{2} r^{2} 10^{-n},
$$

where the summation is over the pairs $\left(m_{1}, m_{2}\right)$ such that the points $\left(m_{1} / 10^{n}, m_{2} / 10^{n}\right)$ belong to the disk mentioned above. It follows that for every ball $B=B(x, r)$ centered at an arbitrary point $x \in \widetilde{Q}$ and of radius $r \leq 20$ we have

$$
\left(A_{3}\right)^{-1} r^{3} \leq \operatorname{Cap}(B(x, r) \backslash K) \leq A_{3} r^{3}
$$

in particular, $(0.2)$ is fulfilled for $B$ and $2 B$. For balls of greater radius and centered at $x \in \widetilde{Q}$, as well as for all balls centered outside $B(0,10)$, inequality (0.2) is obvious (though (1.14) may fail).

Consider a ball $B$ centered at a point belonging to the closure of some set of the form $U\left(m_{1} / 10^{n}, m_{2} / 10^{n}, 1 / 10^{n}\right)$. If $4 B$ intersects the plane $x_{3}=0$, then (0.2) for $B$ and $2 B$ follows from a similar inequality for balls centered in $\widetilde{Q}$. But if $4 B$ does not intersect the plane $x_{3}=0$, then, by construction, $2 B$ does not intersect the closures of the other sets $U\left(m_{1} / 10^{n}, m_{2} / 10^{n}, 1 / 10^{n}\right)$, so, clearly, (0.2) is again satisfied for $B$ and $2 B$.

Thus, (0.2) holds true for $K$.

Consider the function $f=\sigma * E$, where $\sigma$ is a measure uniformly distributed over $\widetilde{Q}$; clearly, $f \in h(K)$. We show that $f \notin H(K)$.

Let $B$ be the ball centered at a point of $\widetilde{Q}$ and of radius $r$; take a function $\varphi$ with $\varphi \equiv 1$ on $(1 / 2) B, \varphi(x) \geq 0$ for all $x$, and $\left\|\nabla^{2} \varphi\right\|_{\mathrm{L}_{\infty}} r^{2} \leq A$. We have

$$
\left|\int_{B} f(x) \Delta \varphi(x) d m_{x}\right|=|\langle\Delta f \mid \varphi\rangle|=\int_{\widetilde{Q}} \varphi\left(x_{1}, x_{2}\right) d x_{1} d x_{2}>\pi r^{2} / 4
$$

and, by (1.14),

$$
\left\|\nabla^{2} \varphi\right\|_{\mathrm{L}_{\infty}} r^{2} \operatorname{Cap}(k B \backslash K) \leq A_{4} k^{3} r^{3} .
$$

This contradicts (0.1) if $r$ is sufficiently small. Now, $f \notin H(K)$ by Lemma 1.3. This finishes the description of the example.

At this point we start the proof of Theorem 11 which will be finished in $\S 3$. In $\S 1$ we obtain a series of estimates and reduce Theorem 1 to Lemma 1.8. By (0.1), for $|\alpha| \geq 0$ we have the following estimates for the coefficients $c_{\alpha}\left(V_{\varphi} f, a\right)$ (see (1.9) ):

$$
\begin{aligned}
\left|c_{\alpha}\left(V_{\varphi} f, a\right)\right| & =(\alpha !)^{-1}\left|\int f(x) \Delta\left(\varphi(x)(x-a)^{\alpha}\right) d m_{x}\right| \\
& \leq(\alpha !)^{-1} A k_{1} \omega_{f}(\delta)\left\|\nabla^{2} \varphi\right\|_{\mathrm{L}_{\infty}}(2 \delta)^{2+|\alpha|} \operatorname{Cap}(k B \backslash X) ;
\end{aligned}
$$


here we have used the elementary estimate

$$
\left\|\nabla^{2}\left(\varphi(x)(x-a)^{\alpha}\right)\right\|_{\mathrm{L}_{\infty}} \leq A_{1}\left\|\nabla^{2} \varphi\right\|_{\mathrm{L}_{\infty}}(2 \delta)^{|\alpha|} .
$$

Lemma 1.4. If $|x-a| \geq 8 \sqrt{3} \delta$, then for every $n \in \mathbb{Z}_{+}$we have

$$
\left|V_{\varphi} f(x)-\sum_{|\alpha|<n} c_{\alpha} \partial^{\alpha} E(x-a)\right| \leq A_{1} k_{1} \omega_{f}(\delta)\left\|\nabla^{2} \varphi\right\|_{\mathrm{L}_{\infty}} \operatorname{Cap}(k B \backslash X) \frac{(7 \sqrt{3} \delta)^{2+n}}{|x-a|^{1+n}}
$$

here $c_{\alpha}=c_{\alpha}\left(V_{\varphi} f, a\right)$. In particular, for $n=0$ we obtain

$$
\left|V_{\varphi} f(x)\right| \leq A_{2} k_{1} \omega_{f}(\delta)\left\|\nabla^{2} \varphi\right\|_{\mathrm{L}_{\infty}} \delta^{2} \frac{\operatorname{Cap}(k B \backslash X)}{|x-a|} .
$$

Proof. By (1.8), (1.15), the estimate

$$
\left|\partial^{\alpha} E(x)\right| \leq \frac{\alpha !(3 \sqrt{3})^{|\alpha|}}{|x|^{1+|\alpha|}}
$$

(see, e.g., [5, Lemma 3.2]), and the fact that the number of $c_{\alpha}$ with $|\alpha|=m$ is $\frac{(m+2)(m+1)}{2}$, we see that the left-hand side of (1.16) does not exceed the quantity

$$
A k_{1} \omega_{f}(\delta)\left\|\nabla^{2} \varphi\right\|_{\mathrm{L}_{\infty}} \operatorname{Cap}(k B \backslash X) \frac{(6 \sqrt{3} \delta)^{2+n}}{|x-a|^{1+n}} \sum_{m=n}^{\infty} \frac{(m+2)(m+1)}{2}\left(\frac{3}{4}\right)^{m-n},
$$

which yields (1.16) immediately.

Remark 1.2. In what follows, we shall localize the function $f$ only with the help of functions $\varphi_{j}$ satisfying the assumptions of Lemma 1.1 (in particular, we always have $\left.\left\|\nabla^{2} \varphi_{j}\right\|_{\mathrm{L}_{\infty}} \leq\left(s\left(Q_{j}\right)\right)^{-2}\right)$; next, (1.16) will be used only for $n \leq 3$.

The estimates of the lemma below are easy generalizations of (1.15) and (1.16), but they do not involve the constant $k$ explicitly. For this reason, they are more convenient in the geometric construction to be presented later on. As before, let $f_{j}=E *\left(\varphi_{j} \Delta f\right)$, let $a_{j}$ be the center of $Q_{j}$, and let $s_{j}=s\left(Q_{j}\right)$.

Lemma 1.5. Under the assumptions of Lemma 1.1, there exists a partition of unity $\left\{\varphi_{j}\right\}$ for $\bigcup_{j} Q_{j}$ such that $\operatorname{Spt} \varphi_{j} \subset(9 / 8) Q_{j}$ and $\left\|\nabla^{3} \varphi_{j}\right\|_{\mathrm{L}_{\infty}} \leq A\left(s_{j}\right)^{-3}$.

Moreover, the corresponding localizations $f_{j}$ obey the estimates

(1) $\left|c_{\alpha}\left(f_{j}, a_{j}\right)\right| \leq k_{2} \omega_{f}\left(s_{j}\right) \operatorname{Cap}\left((5 / 4) Q_{j}^{\circ} \backslash X\right)\left(s_{j}\right)^{|\alpha|}, 0 \leq|\alpha| \leq 2$;

$$
\left|f_{j}(x)-\sum_{|\alpha|<n} c_{\alpha}\left(f_{j}, a_{j}\right) \partial^{\alpha} E\left(x-a_{j}\right)\right| \leq k_{2} \omega_{f}\left(s_{j}\right) \operatorname{Cap}\left((5 / 4) Q_{j}^{\circ} \backslash X\right) \frac{\left(s_{j}\right)^{n}}{\left|x-a_{j}\right|^{1+n}},
$$

for $x \notin(5 / 4) Q_{j}$ and $n \leq 3$, where the constant $k_{2}$ depends only on $k$ and $k_{1}$ in (0.1).

Proof. We decompose every cube $Q_{j}$ into dyadic cubes $Q_{j, p}$ with side length $2^{-N} s_{j}$, where $N=N(k) \in \mathbb{N}$ is the minimal number with the property that for the balls $B_{j, p}$ centered at $a_{j, p}$ and circumscribed around $(3 / 2) Q_{j, p}$ we have $(8 \sqrt{3}) B_{j, p} \subset(9 / 8) Q_{j}$ and $k B_{j, p} \subset$ $(9 / 8) Q_{j}$, where $k$ is as in (0.1). Applying Lemma 1.1 to the cubes $Q_{j, p}$, we construct a partition of unity $\left\{\varphi_{j, p}\right\}$ for $Q_{j}$ (with appropriate estimates for the derivatives) and the localizations $f_{j, p}=E *\left(\varphi_{j, p} \Delta f\right)$.

Take $\varphi_{j}=\sum_{p} \varphi_{j, p}$ and $f_{j}=\sum_{p} f_{j, p}$. Using Remark 1.1, we expand every $f_{j, p}$ in a Laurent series centered at $a_{j}$, and in another Laurent series centered at $a_{j, p}$ (the expansions represent the function outside the corresponding balls). Much as we have deduced (0.1) from (1.15), we can show that

$$
\left|c_{\alpha}\left(f_{j, p}, a_{j}\right)\right| \leq k_{1} \omega_{f}\left(s_{j}\right) \operatorname{Cap}\left(k B_{j, p} \backslash X\right)\left(s_{j}\right)^{|\alpha|}, \quad 0 \leq|\alpha| \leq 2 .
$$


Since Spt $\varphi_{j, p} \subset B_{j, p}$ and $k B_{j, p} \subset(9 / 8) Q_{j}$, we obtain (1) with $c_{\alpha}\left(f_{j}, a_{j}\right)=\sum_{p} c_{\alpha}\left(f_{j, p}, a_{j}\right)$.

For $n=0$, (1.17) is a consequence of (1.16) for $f_{j, p}$. If $n>0$, it suffices to establish estimates similar to (1.17) with $f_{j}$ in place of $f_{j, p}$. By (1.16) it suffices to show the following for the Laurent expansion of $f_{j, p}$ centered at $a_{j, p}$ : if $x \notin(5 / 4) Q_{j}$ and $n \leq 3$, then

$$
\begin{aligned}
& \left|\sum_{|\alpha|<n}\left(c_{\alpha}\left(f_{j, p}, a_{j}\right) \partial^{\alpha} E\left(x-a_{j}\right)-c_{\alpha}\left(f_{j, p}, a_{j, p}\right) \partial^{\alpha} E\left(x-a_{j, p}\right)\right)\right| \\
& \quad \leq k_{1} \omega_{f}\left(s_{j}\right) \operatorname{Cap}\left(k B_{j, p} \backslash X\right) \frac{\left(s_{j}\right)^{n}}{\left|x-a_{j}\right|^{1+n}} .
\end{aligned}
$$

But (1.19) follows from (1.18) and (1.15) for $c_{\alpha}\left(f_{j, p}, a_{j, p}\right)$ and from the asymptotics of the left-hand side of (1.19) at infinity.

Recall that Vitushkin's method allows us to reduce the approximation problem in question to the construction of functions that equate a due set of Laurent coefficients for localizations. We look more carefully at the question as to how many (and which particular) coefficients should be involved.

We shall need a particular case of Theorem 2 in 8 .

Lemma 1.6. Suppose that there exists a constant $C=C(f, X)>0$ with the following property. For every covering $\left\{Q_{j}\left(a_{j}, s_{j}\right)\right\}$ of the compact set $\operatorname{Spt}(\Delta f)$ by a finite collection of disjoint dyadic cubes and for every corresponding localization $f_{j}$ with the function $\varphi_{j}$ given by Lemma 1.1, there exists a function $\Phi_{j}$ such that $\Phi_{j} \in C\left(\mathbb{R}^{3}\right), \lim _{x \rightarrow \infty} \Phi_{j}(x)=0$, and

(1) $\operatorname{Spt}\left(\Delta \Phi_{j}\right) \subset\left(C Q_{j}^{\circ} \backslash X\right)$;

Then $f \in H(X)$.

$$
\left|f_{j}(x)-\Phi_{j}(x)\right| \leq C \omega_{f}\left(s_{j}\right) \min \left(1, \frac{\left(s_{j}\right)^{3}}{\left|x-a_{j}\right|^{3}}\right) .
$$

Let us compare Lemmas 1.6 and 1.5. By condition (1) in Lemma 1.5 with $\alpha=0$, the definition of the capacity shows that for every $j$ there exists $\Phi_{j} \in C\left(\mathbb{R}^{3}\right)$ with $\lim _{x \rightarrow \infty} \Phi_{j}(x)=0$ that satisfies condition (1) in Lemma 1.6 with $C=5 / 4$ and has the properties $\left\|\Phi_{j}\right\|_{\mathrm{L}_{\infty}} \leq k_{2} \omega_{f}\left(s_{j}\right)$ and $c_{0}\left(f_{j}\right)=c_{0}\left(\Phi_{j}\right)$ (Remark 1.1 is applicable to $\left.\Phi_{j}\right)$. By Lemma 1.2 and condition (2) in Lemma 1.5, this leads to the estimate

$$
\left|f_{j}(x)-\Phi_{j}(x)\right| \leq A_{1} k_{2} \omega_{f}\left(s_{j}\right) \min \left(1, \frac{\left(s_{j}\right)^{2}}{\left|x-a_{j}\right|^{2}}\right),
$$

which is weaker than the required estimate (1.20) (the power order on the right is smaller by 1$)$.

The next statement is a reformulation of Lemma 1.6

Lemma 1.7. Under the assumptions of Lemma 1.6, suppose that there exists a constant $C=C(f, X)>0$ and a function $\Phi_{j} \in C\left(\mathbb{R}^{3}\right), \lim _{x \rightarrow \infty} \Phi_{j}(x)=0$, such that

(1) $\operatorname{Spt}\left(\Delta \Phi_{j}\right) \subset\left(C Q_{j}^{\circ} \backslash X\right)$;

(2) $\left\|\Phi_{j}\right\|_{L_{\infty}} \leq C \omega_{f}\left(s_{j}\right)$;

(3) $c_{\alpha}\left(f_{j}, a_{j}\right)=c_{\alpha}\left(\Phi_{j}, a_{j}\right)|\alpha| \leq 1$.

Then $f \in H(X)$.

So, in order to prove Theorem 1, it suffices to ensure the assumptions of Lemma 1.7. Furthermore, since (3) for $\alpha=0$ follows directly from (1.21) (in fact, from (0.1)), it suffices to ensure the relation $c_{\alpha}\left(f_{j}, a_{j}\right)=c_{\alpha}\left(\Phi_{j}, a_{j}\right)$ with arbitrary $j$ only for $|\alpha|=1$. 
Remark 1.3. As was mentioned in [8], in the case of uniform approximation in the class of analytic functions, there is no "gap of magnitude 1 in the order" between an analog of (0.1) (see [3, Chapter 4, §2, Lemma 1]) and the requirements of Theorem 2 in [8]. Therefore, Vitushkin's criterion is a direct consequence of that theorem. In essence, the situation is similar in the case of harmonic approximation in the $C^{1}$-norm. To prove Theorem 1 (more generally, Conjecture 1), we must equate additionally three coefficients $c_{\alpha}$ with $|\alpha|=1$ for the localizations of the initial function. Should we have chosen to mimic the proof of Lemma 1 in [3. Chapter 2, §4] instead of applying Lemma 1.7, this would have required a much harder prerequisite, specifically, an estimate like (1.20) but with the fourth power on the right instead of the third. This would have led to the task of equating six more coefficients $c_{\alpha}$ with $|\alpha|=2$, which would have produced substantial complications at least.

We continue the proof of Theorem 1 Let us simplify the notation.

In what follows, the coefficients of $\left(\partial / \partial x_{m}\right) E$ in a Laurent expansion will be denoted by $c_{m}^{1}, m=1,2,3$. By (1.21), we may assume at once that $c_{0}\left(f_{j}\right)=0$ and, consequently, $f_{j}(x)=O\left(|x|^{-2}\right)$ as $x \rightarrow \infty$. Since Lemma 1.7 is about localizations, we fix a localization $f_{j}$ and denote it by $g$. Making (if necessary) a shift and a homothety (which preserve (0.1) and (0.2) with the same constants), we may assume that $\operatorname{Spt}(\Delta g) \subset(1 / 2) \mathbf{Q}_{0}$, where $\mathbf{Q}_{0}=[0,1]^{3}$ is the unit cube. To lighten the notation, we still use the symbol $X$ to denote the transform of the initial compact set. Also, we shall assume that $\|g\|_{\mathrm{L}_{\infty}} \leq 1$.

The main ingredient in the deduction of Theorem 1 from Lemma 1.7 is the following Lemma 1.8. This is a statement about equating the coefficient $c_{3}^{1}$ (one among the three we require).

Lemma 1.8. Suppose that $g \in C\left(\mathbb{R}^{3}\right)$ satisfies $\operatorname{Spt}(\Delta g) \subset\left((1 / 2) \mathbf{Q}_{0} \backslash X^{\circ}\right)$ and condition (0.2) is fulfilled; suppose also that (0.1) is true with $g$ in place of $f$. Also, we assume that $g(x)=O\left(|x|^{-2}\right)$ as $x \rightarrow \infty$ and $\|g\|_{\mathrm{L}_{\infty}} \leq 1$.

Then there exist functions $G_{3}^{1}, G_{3}^{2} \in C\left(\mathbb{R}^{3}\right)$ with the following properties:

(1) $\operatorname{Spt}\left(\Delta G_{3}^{p}\right) \subset\left(2 \mathbf{Q}_{0} \backslash X\right)$ and $G_{3}^{p}(x)=O\left(|x|^{-2}\right)$ as $x \rightarrow \infty(p=1,2)$;

(2) $\left\|G_{3}^{1}\right\|_{\mathrm{L}_{\infty}} \leq k_{3}$ and $\left\|G_{3}^{2}\right\|_{\mathrm{L}_{\infty}} \leq k_{4}$, where $k_{3}$ depends on $k_{2}$ in Lemma 1.5, and $k_{4}$ depends on $k_{2}$ and on $k_{0}$ in (0.2);

(3) $c_{3}^{1}\left(G_{3}^{2}\right) \geq \sum_{j=1}^{3}\left|c_{j}^{1}\right|\left(g-G_{3}^{1}\right)$;

(4) $c_{3}^{1}\left(G_{3}^{2}\right) \geq(1 / 8) \max _{j \neq 3}\left|c_{j}^{1}\right|\left(G_{3}^{2}\right)$.

Remark 1.4. Formally, Lemma [1.8 is similar to Lemma 1.4 in [9] or Lemma 3.7 in [8], but it is much more complicated in essence. It should be noted that the dependence of $k_{4}$ in (2) on $k_{0}$ in (0.2) is the only obstruction to a complete proof of Conjecture 1 .

Theorem 1 follows from Lemma 1.7 and Lemma 1.8, which is a generalization of Lemma 1.8 .

Lemma 1.8. Let $g$ be as in Lemma 1.8. Then there exist 6 functions $G_{m}^{p} \in C\left(\mathbb{R}^{3}\right)$ $(m=1,2,3, p=1,2)$ such that

(1) $\operatorname{Spt}\left(\Delta G_{m}^{p}\right) \subset\left(2 \mathbf{Q}_{0} \backslash X\right)$ and $G_{m}^{p}(x)=O\left(|x|^{-2}\right)$ as $x \rightarrow \infty$;

(2) $\left\|G_{m}^{1}\right\|_{\mathrm{L}_{\infty}} \leq k_{3},\left\|G_{m}^{2}\right\|_{\mathrm{L}_{\infty}} \leq k_{4}$;

(3) $c_{m}^{1}\left(G_{m}^{2}\right) \geq \sum_{j=1}^{3}\left|c_{j}^{1}\right|\left(g-G_{m}^{1}\right)$;

(4) $c_{m}^{1}\left(G_{m}^{2}\right) \geq 2 \max _{j \neq m}\left|c_{j}^{1}\right|\left(G_{m}^{2}\right)$.

Indeed, using Lemma 1.8, it is easy to ensure the assumptions of Lemma 1.7, and this suffices for the proof of Theorem 1. Let $m_{0}$ be the index such that the sum $\sum_{j=1}^{3}\left|c_{j}^{1}\right|\left(g-G_{m}^{1}\right)$ is minimal among all $m$. Then a function equating all coefficients $c_{\alpha}$ in the expansion of $g$ can be found in the form $G_{m_{0}}^{1}+\sum_{m=1}^{3} t_{m} G_{m}^{2}$; moreover, $\left|t_{m}\right| \leq A$ 
for all $m$ by condition (4) of Lemma 1.8, because the linear system determining the $t_{m}$ has a matrix with dominating diagonal.

We outline the further steps of the proof of Theorem 1. Below, in $\S \S 1-3$, we prove Lemma 1.8. After that (at the end of $\S 3$ ), we shall show how to prove Lemma 1.8 by an easy modification of the arguments. This will finish the proof of Theorem 1 .

Proof of Lemma 1.8. We assume that $g \not \equiv 0$ (otherwise there is nothing to prove). In the rest of $\S 1$, we introduce some definitions and notation, obtain some estimates, and indicate the form in which we shall construct the function $G_{3}^{2}$. In $\S 3$ we present a geometric construction and reduce Lemma 1.8 to the technical Lemma 2.3. The latter lemma will be proved in $\S 3$.

Consider a covering of $\mathbf{Q}_{0}$ by disjoint dyadic cubes (it will be specified in $\S \S 2-3$ ) and construct the associated partition of unity in accordance with Lemma 1.5.

Let $g_{j}$ be the corresponding localizations of $g$ (they are second order localizations for the initial function $f)$. By Lemma 1.5, there exist functions $\Phi_{j} \in C\left(\mathbb{R}^{3}\right)$ with $\operatorname{Spt}\left(\Delta \Phi_{j}\right) \subset\left((5 / 4) Q_{j}^{\circ} \backslash X\right)$ such that $c_{0}\left(g_{j}\right)=c_{0}\left(\Phi_{j}\right)$ and the functions $r_{j}=g_{j}-\Phi_{j}$ satisfy the following estimate (here $a_{j}$ is the center of $Q_{j}, s_{j}=s\left(Q_{j}\right)$, and $\beta_{j}=\beta\left(Q_{j}\right)=$ $\left.\operatorname{Cap}\left((5 / 4) Q_{j}^{\circ} \backslash X\right)\right)$ :

$$
\left\|r_{j}\right\|_{\mathrm{L}_{\infty}} \leq A k_{2} \omega_{g}\left(s_{j}\right) ; \quad\left|r_{j}(x)\right| \leq A k_{2} \omega_{g}\left(s_{j}\right) \frac{s_{j} \beta_{j}}{\left|x-a_{j}\right|^{2}} \quad \text { for } \quad x \notin(3 / 2) Q_{j} .
$$

In should be noted that, if the capacity $\beta_{j}$ is small compared to $s_{j}$, then the estimate for $\left|r_{j}(x)\right|$ is "much worse" for $x \in Q_{j}$ than for $x \notin(3 / 2) Q_{j}$; this explains condition (4) in Lemma 2.1).

We refine the asymptotic estimate for $r_{j}$ if $x \notin(3 / 2) Q_{j}$. By Lemma 1.5, we have

$$
\begin{aligned}
\left|r_{j}(x)-\sum_{m=1}^{3} c_{m}^{1}\left(r_{j}\right) \frac{\partial E\left(x-a_{j}\right)}{\partial x_{m}}\right| & \leq A k_{2} \omega_{g}\left(s_{j}\right) \frac{\left(s_{j}\right)^{2} \beta_{j}}{\left|x-a_{j}\right|^{3}} ; \\
\sum_{m=1}^{3}\left|c_{m}^{1}\left(r_{j}\right)\right| & \leq A k_{2} \omega_{g}\left(s_{j}\right) s_{j} \beta_{j} .
\end{aligned}
$$

The function $G_{3}^{2}$ will be constructed as a finite linear combination with positive coefficients of functions $h_{2,1}$ as in (1.26) below. We start with several definitions.

A column is any set of dyadic cubes (1.7) such that $p, m_{1}$ and $m_{2}$ are fixed (the cubes involved are of one and the same size and are placed in the direction of the $x_{3}$-axis). Two different columns $\left\{Q_{p}^{m_{1}, m_{2}, m_{3}}\right\}$ and $\left\{Q_{p}^{m_{1}^{\prime}, m_{2}^{\prime}, m_{3}^{\prime}}\right\}$ are said to be neighboring if $\max \left(\left|m_{1}-m_{1}^{\prime}\right|,\left|m_{2}-m_{2}^{\prime}\right|\right)=1$.

Two dyadic cubes are said to be coordinated (we also use the term "a coordinated pair of cubes") is they are of equal size, they belong to one and the same column or to two neighboring columns, and the $m_{3}$-indices of these cubes (see (1.7)) differ by 2 or 3 . In what follows, we always assume that $m_{3}\left(Q_{2}\right)>m_{3}\left(Q_{1}\right)\left(Q_{2}\right.$ is placed "higher" than $\left.Q_{1}\right)$.

Let $\left(Q_{1}, Q_{2}\right)$ be a coordinated pair such that the two sets $(9 / 8) Q_{j} \backslash X^{\circ}$ with $j=1,2$ are nonempty. By (0.2), we have

$$
\max \left(\beta_{1}, \beta_{2}\right) \leq k_{5} \min \left(\beta_{1}, \beta_{2}\right),
$$

where $\beta_{j}=\operatorname{Cap}\left((5 / 4) Q_{j}^{\circ} \backslash X\right)(j=1,2)$. Here the two quantities $\beta_{j}$ are positive and $k_{5} \geq 1$ depends only on $k_{0}$ in (0.2). (It should be noted that, if (0.2) fails, i.e., in the general case of Conjecture 1 the situation becomes substantially complicated.)

By the definition of capacity, there exist $h_{j} \in C\left(\mathbb{R}^{3}\right)(j=1,2)$ of the form $h_{j}=$ $E * \mu_{j}$, where the $\mu_{j}$ are nonnegative measures with $\operatorname{Spt}\left(\mu_{j}\right) \subset\left((5 / 4) Q_{j}^{\circ} \backslash X\right)$ such that 
$\left\|h_{j}\right\|_{\mathrm{L}_{\infty}} \leq(2 \pi)^{-1}$ and $\left\|\mu_{j}\right\|=c_{0}\left(h_{j}\right)=\beta_{j}$ (observe the normalization in (1.1) and (1.3), and Remark 1.1). Consider the function

$$
h_{2,1}=\lambda_{2} h_{2}-\lambda_{1} h_{1},
$$

where the constants $\lambda_{1}$ and $\lambda_{2}$ are defined as follows: if $\beta_{2} \leq \beta_{1}$, we take $\lambda_{1}=\lambda_{1}\left(Q_{1}\right)=$ $\beta_{2} / \beta_{1}$ and $\lambda_{2}=\lambda_{2}\left(Q_{2}\right)=1$, and if $\beta_{2}>\beta_{1}$, we take $\lambda_{2}=\beta_{1} / \beta_{2}$ and $\lambda_{1}=1$. Clearly, under this choice we have

$$
c_{0}\left(h_{2,1}\right)=0, \quad \lambda_{1} \beta_{1}=\lambda_{2} \beta_{2}=\min \left(\beta_{1}, \beta_{2}\right),
$$

and the coefficients $c_{j}^{1}(j=1,2,3)$ (the senior Laurent coefficients of $\left.h_{2,1}\right)$ do not depend on the center of the Laurent expansion.

By (1.25), in any case the coefficients $\lambda_{1}$ and $\lambda_{2}$ in the definition of $h_{2,1}$ satisfy the estimate

$$
\frac{1}{k_{5}} \leq \min \left(\lambda_{1}, \lambda_{2}\right) \leq \max \left(\lambda_{1}, \lambda_{2}\right)=1
$$

Let $s=s\left(Q_{1}\right)=s\left(Q_{2}\right)$; the definition of coordinated pairs shows that

$$
c_{3}^{1}\left(h_{2,1}\right) \geq(s / 2) \min \left(\beta_{1}, \beta_{2}\right), \quad \max _{j=1,2}\left|c_{j}^{1}\right|\left(h_{2,1}\right) \leq 4 s \min \left(\beta_{1}, \beta_{2}\right),
$$

in particular, condition (4) of Lemma 1.8 is fulfilled. (Indeed, since the $h_{j}$ are obtained by convolving $E$ with nonnegative measures, (1.29) is a consequence of a similar (and obvious) estimate for the function $h_{2,1}=\lambda_{2} \beta_{2} E\left(x-b_{2}\right)-\lambda_{1} \beta_{1} E\left(x-b_{1}\right)$, where the $b_{j}$ $(j=1,2)$ are arbitrary points of the dilations $(5 / 4) Q_{j}$ for the cubes of the coordinated pair in question.)

The functions $h_{2,1}$ are estimated much as the $r_{j}$ in (1.22). By condition (2) of Lemma 1.5, for $n=1$ we have

$$
\left|h_{2,1}(x)\right| \leq A_{1} \frac{s \min \left(\beta_{1}, \beta_{2}\right)}{\left|x-a_{1}\right|^{2}} \quad \text { for } \quad x \notin\left((3 / 2) Q_{1} \cup(3 / 2) Q_{2}\right)
$$

( $a_{1}$ is the center of $Q_{1}$; it can be replaced by the center of the other cube in the coordinated pair in question).

\section{$\S 2$. Construction. An outline of the proof of Lemma 1.8}

Lemma 2.1. Let $X$ be a compact set satisfying (0.2), and let $g$ be the function mentioned in Lemma 1.8. Then there exists a finite family Cover of disjoint dyadic cubes that covers $(1 / 2) \mathbf{Q}_{0} \backslash X^{\circ}$, a finite collection Pair of coordinated pairs $\left(Q_{1}, Q_{2}\right)$ of cubes (one and the same cube may belong to different pairs), and the corresponding collection $\mathbf{H}(\mathbf{P a i r})$ of functions $h_{2,1}$ as in (1.26) such that the following conditions are satisfied.

(1) Position of cubes. There exists a Lipschitz surface $\Gamma$ determined by the equation $x_{3}=\Psi\left(x_{1}, x_{2}\right)$, where $\left(x_{1}, x_{2}\right) \in[0,1]^{2}$, such that $|\nabla \Psi| \leq A$ and for every cube $Q$ in Cover we have $\operatorname{dist}(Q, \Gamma) \leq A_{1} s(Q)$. Furthermore, every cube belonging to some pair in Pair includes (or, maybe, coincides with) some cube in Cover.

(2) Estimate of $c_{3}^{1}$. Let $C_{\text {cover }}$ denote the sum of $\beta(Q) s(Q)$ over all cubes $Q$ that occur in some pair in Pair. Then the sum of $\beta(Q) s(Q) \omega_{g}(s(Q))$ over all cubes in Cover does not exceed $k_{6} C_{\text {cover }}$, where $k_{6}$ depends only on $k_{0}$ in (0.2).

(3) Carleson condition. Let $Q^{\prime}$ be an arbitrary dyadic cube. Then the sum of $\beta(Q) s(Q)$ over all cubes in Cover that are included in $Q^{\prime}$ and belong to some pair in Pair does not exceed $A_{2}\left(s\left(Q^{\prime}\right)\right)^{2}$.

(4) Bounded multiplicity of intersection for dilated cubes. The sum of the quantities $\chi((3 / 2) Q)(x)$ over all $Q \in$ Cover and the sum of $\chi((3 / 2) Q)(x)$ over all $Q$ occurring in some pair in Pair does not exceed $A_{3}(\chi(\cdot)$ stands for a characteristic function). 
Remark 2.1. Condition (2) in Lemma 2.1 and formulas (1.28) and (1.29) imply that the sum of $\beta(Q) s(Q) \omega_{g}(s(Q))$ over all cubes in Cover does not exceed $2 k_{5} k_{6}$ times the sum of $c_{3}^{1}\left(h_{2,1}\right)$ over all $h_{2,1} \in \mathbf{H}($ Pair $)$.

Remark 2.2. The construction that provides the assumptions of the lemma consists of two steps: the main part and the supplementary part. In the main part, we shall construct a cover Cover $_{0}$ and a finite collection Pair $_{0}$ of coordinated pairs that satisfy conditions (1)-(3) of Lemma 2.1. In the supplementary part, we describe an additional construction that ensures also condition (4) in Lemma 2.1. The family Cover will be obtained from Cover $_{0}$ by splitting certain cubes of Cover $_{0}$ into smaller cubes; Pair will be obtained from Pair $_{0}$ by dropping certain coordinated pairs of cubes. We shall retain control over the changes of the constants under these procedures.

Proof of Lemma 2.1. Construction: the main part. We construct by induction a "nondecreasing" sequence $\left\{Q_{j}\right\}(p)$ of coverings, $p=0,1,2, \ldots$, where all $Q_{j}$ are dyadic cubes. We take $\left\{Q_{j}\right\}(0)=\mathbf{Q}_{0}$; every cube of $\left\{Q_{j}\right\}(p+1)$ is included in some cube of $\left\{Q_{j}\right\}(p)$ (these cubes may coincide); the index $p$ at which the process stops will be indicated below. Each covering consists of white and red cubes. White cubes undergo splitting at each step except the last; red cubes are fixed at each step and are collected without further splitting (i.e., they retain the color). The side length of every white cube in $\left\{Q_{j}\right\}(p)$ is $2^{-p}$, the side length of every red cube in the same cover is at least $2^{-p}$.

Base of induction: $\left\{Q_{j}\right\}(0)=\mathbf{Q}_{0}$, and $\mathbf{Q}_{0}$ is white.

Induction step: construction of $\left\{Q_{j}\right\}(p+1)$. We split each white cube $Q$ in $\left\{Q_{j}\right\}(p)$ into 8 dyadic cubes of equal size, i.e., with edge length $s(Q) / 2=2^{-p-1}$. We attribute the resulting cubes to the $(p+1)$ st generation. Among them, we attribute to $\left\{Q_{j}\right\}(p+1)$ only those cubes $Q_{j}$ for which $(9 / 8) Q_{j} \backslash X^{\circ}$ is nonempty (recall that otherwise $g_{j} \equiv 0$, where $g_{j}$ is the localization from Lemma 1.5). Thus, $\left\{Q_{j}\right\}(p+1)$ consists of cubes of the $(p+1)$ st generation that have the above property and the set (maybe, empty) of all red cubes of the cover $\left\{Q_{j}\right\}(p)$.

It remains to declare some cubes of the $(p+1)$ st generation red; the other cubes will be declared white. Let $Q$ belong to the $(p+1)$ st covering $\left\{Q_{j}\right\}(p+1)$; if some cube $Q^{\prime}$ in the same covering is coordinated with $Q$, we fix and declare red every cube of $\left\{Q_{j}\right\}(p+1)$ placed in the same column as $Q$ and $Q^{\prime}$ (in particular, $Q$ and $Q^{\prime}$ themselves are red); next, we construct the function $h_{2,1}$ in accordance with (1.26) by the pair $\left(Q, Q^{\prime}\right)$. This finishes the description of $\left\{Q_{j}\right\}(p+1)$.

It can easily be checked by induction that for every $p$ the cover $\left\{Q_{j}\right\}(p)$ possesses the following properties (a) and (b).

(a) All cubes of the cover $\left\{Q_{j}\right\}(p)$ are divided into groups in such a way that every group is situated in it own column, all cubes in one group are of the same color, and the projections of these groups to the plane $x_{3}=0$ have mutually disjoint interiors. Furthermore, the indices $m_{3}$ (see (1.7) ) may differ at most by 3 within one group of cubes; if all cubes in the group are white, the indices may differ at most by 1 (i.e., the cubes are neighboring).

(Indeed, at most 2 neighboring white cubes in a column may undergo splitting, which results in the arising of at most 4 cubes in a column for the next generation, and the indices $m_{3}$ for these cubes may differ at most by 3 . Some of these cubes are included in the next covering, and the other are dropped; by the definition of coordinated pairs, either all cubes in the column that are included in the covering are declared red and are fixed, or at most two white cubes are included in the covering.)

(b) Let $Q$ and $Q^{\prime}$ be $p$ th generation cubes that belong to $\left\{Q_{j}\right\}(p)$ and are situated in neighboring columns. Then their indices $m_{3}$ differ at most by 3 ; if the cubes are white, the indices differ at most by 1 . 
(Indeed, two cubes of an earlier generation that contain $Q$ and $Q^{\prime}$ would have been fixed as coordinated.)

Among other things, property (a) implies that the sum of $\beta_{j} s\left(Q_{j}\right) \omega_{g}\left(s\left(Q_{j}\right)\right)$ over all white cubes in $\left\{Q_{j}\right\}(p)$ does not exceed $A \omega_{g}\left(2^{-p}\right)$ (indeed, the side length of a white cube is $2^{-p}$, each column contains at most 2 white cubes, and their projections to the plane $x_{3}=0$ are mutually disjoint, so that there are at most $2^{2 p+1}$ white cubes in $\left\{Q_{j}\right\}(p)$; at the same time, $\left.\beta_{j} s\left(Q_{j}\right) \leq A_{1} 2^{-2 p}\right)$.

Since $\operatorname{Spt}(\Delta g) \subset(1 / 2) \mathbf{Q}_{0}$ and $g \not \equiv 0$, we see that $\mathbf{Q}_{0}{ }^{\circ} \backslash X$ is a nonempty open set; therefore, it cannot be that all coverings $\left\{Q_{j}\right\}(p)$ consist entirely of white cubes. This enables us to define the last index $p$.

Stopping time. The covering $\left\{Q_{j}\right\}(p)$ is fixed if at least one of the following two conditions is fulfilled: all cubes are red (then there is nothing to split further) or $\omega_{g}\left(2^{-p}\right)$ is smaller than the sum of $\beta(Q) s(Q)$ over all red cubes of $\left\{Q_{j}\right\}(p)$.

So, we fix the number $p$ indicated above, put Cover $_{0}=\left\{Q_{j}\right\}(p)$, and define Pair $_{0}$ to be the collection of all coordinated pairs of red cubes and $\mathbf{H}\left(\mathbf{P a i r}_{0}\right)$ to be the set of the corresponding functions $h_{2,1}$.

In accordance with property (a), we choose arbitrarily one cube in each column of Cover $_{0}$. Let $Q$ and $Q^{\prime}$ be chosen in different columns, and let $a\left(x_{1}, x_{2}, x_{3}\right)$ and $a^{\prime}\left(x_{1}^{\prime}, x_{2}^{\prime}, x_{3}^{\prime}\right)$ be their centers. Then

$$
\left|x_{3}-x_{3}^{\prime}\right| \leq 3\left(\left|x_{1}-x_{1}^{\prime}\right|+\left|x_{2}-x_{2}^{\prime}\right|\right) \text {. }
$$

(Indeed, let $p_{1} \leq p$ be the maximal number with the property that the $p_{1}$ th generation cubes $Q_{1}$ and $Q_{1}^{\prime}$ including $Q$ and $Q^{\prime}$ are in neighboring columns. We know that $Q_{1}$ and $Q_{1}^{\prime}$ satisfy (b). Next, since $p_{1}$ is maximal, either one of $Q_{1}$ and $Q_{1}^{\prime}$ was fixed, or the two were white in $\left\{Q_{j}\right\}\left(p_{1}\right)$. In the latter case the $\left(p_{1}+1\right)$ st generation cubes including $Q$ and $Q^{\prime}$ are no longer in neighboring columns. Geometric considerations imply (2.1) in either case.)

We project the selected cubes to the plane $x_{3}=0$. By (2.1), the $x_{3}$-coordinates of the centers of these cubes determine a Lipschitz function $x_{3}=\Psi\left(x_{1}, x_{2}\right)$ on the projection of the set of centers; moreover, $|\nabla \Psi| \leq 3$. By the Whitney theorem (see 6. Chapter 6 , $\S 2$, Theorem 3]), the function $\Psi$ admits an extension to $[0,1]^{2}$ satisfying the condition $|\nabla \Psi| \leq A$. We take the graph of this extension for the role of $\Gamma$.

Now, we show that conditions (1)-(3) of Lemma 2.1 are satisfied.

Condition (1) follows from (a) and formula (2.1). Furthermore, $A_{1}=5 / 2$ because the $m_{3}$-indices of cubes in one and the same column of Cover $_{0}$ differ at most by 3 ; also, every cube belonging to a pair in $\mathbf{P a i r}_{0}$ coincides with a cube in $\mathbf{C o v e r}$.

Condition (3) is also a consequence of (a), by the elementary estimate $\beta(Q) \leq 2 s(Q)$.

We denote by $C_{\text {cover }}^{0}$ the sum of $\beta(Q) s(Q)$ over all cubes belonging to some pair in Pair $_{0}$. For red cubes, condition (2) (with $C_{\text {cover }}^{0}$ in place of $C_{\text {cover }}$ ) follows from (a) (the $m_{3}$-indices within a column differ at most by 3 ) and (0.2). Indeed, these two facts show that the capacity $\beta(Q)$ of a red cube not belonging to any coordinated pair in $\mathbf{P a i r}_{0}$ is dominated by the capacity of some cube lying in the same column and belonging to some coordinated pair. For white cubes in $\mathbf{C o v e r}_{0}$, condition (2) follows from the stopping time definition.

This finishes the main part of the construction.

Construction: supplementary part. This part is aimed at ensuring condition (4) of Lemma 2.1. To do this, we split further some cubes in $\mathbf{C o v e r}_{0}$ and remove some pairs from $\mathbf{P a i r}_{0}$ along with the corresponding functions $h_{2,1}$ in $\mathbf{H}\left(\mathbf{P a i r}_{0}\right)$.

We recall Whitney's well-known construction (see, e.g., [6, Chapter 6, §1]). 
Let $F$ be a compact subset of $\mathbb{R}^{3}$. Then there exists a decomposition of the complement of $F$ in a disjoint union of dyadic cubes $D_{j}$ with the following properties:

(i) $\sqrt{3} s\left(D_{j}\right) \leq \operatorname{dist}\left(D_{j}, F\right) \leq 4 \sqrt{3} s\left(D_{j}\right)$;

(ii) if two cubes in $\left\{D_{j}\right\}$ have a boundary point in common, then the ratio of their side lengths is at most 4.

(Clearly, the intersection multiplicity for the cubes $(3 / 2) D_{j}$ does not exceed a universal constant.)

As $F$, we take the "frame of $\Gamma$ ", that is, the set obtained as the intersection of $\Gamma$ with the lateral faces of all cubes in Cover $_{0}$.

Next, we proceed as follows. We replace each cube $Q$ in Cover $_{0}$ by the Whitney cubes for $F$ included in it if such cubes exist; otherwise, $Q$ remains intact. In the resulting family, we replace every cube with edge length smaller than $2^{-p}$ by a bigger cube with edge length $2^{-p}$, after which we remove all cubes included in $X^{\circ}$. The result is the required cover denoted by Cover in Lemma 2.1. Observe that a cube belonging to a pair in Pair $_{0}$ may fail to coincide with a cube in Cover, but it includes such a cube.

By the properties of Whitney's cubes, the cubes in Cover satisfy conditions (1) and (4) of Lemma 2.1. To verify condition (2) of Lemma 2.1 for Cover and Pair ${ }_{0}$, it suffices to show that the sum of $\beta(Q) s(Q) \omega_{g}(s(Q))$ over all cubes in Cover is at most $A$ times the same sum over Cover $_{0}$. This will follow from a corollary to Lemma 2.2

Lemma 2.2. Let $Q$ be a dyadic cube, and let $\left\{Q_{j}\right\}$ be an at most countable collection of (possibly, intersecting) cubes with $Q_{j} \subset Q$. Let $\widetilde{Q}$ and $\widetilde{Q}_{j}$ be the projections of $Q$ and $Q_{j}$ to the plane $x_{3}=0$.

(1) Suppose that $\lambda_{j}=\lambda\left(Q_{j}\right) \in(0,1]$ satisfy

$$
\sum_{j} \lambda_{j} \chi\left(\widetilde{Q}_{j}\right)(x) \leq 1
$$

for all $x \in \widetilde{Q}$, where $\chi(\cdot)$ is a characteristic function. Suppose that $t_{j} Q_{j} \subset t Q$ with some $t_{j} \in[1,2]$ for all $j, t \geq 1$. Then

$$
\sum_{j} \lambda_{j} \operatorname{Cap}\left(t_{j} Q_{j}^{\circ} \backslash X\right) s\left(Q_{j}\right) \leq A \operatorname{Cap}\left(t Q^{\circ} \backslash X\right) s(Q) .
$$

(2) If, moreover, all $Q_{j}$ are of the same size $\left(s(Q) / s\left(Q_{j}\right)=2^{m}\right.$ with $\left.m \geq 1\right)$ and $\operatorname{dist}\left(\widetilde{Q}_{j}, \partial \widetilde{Q}\right) \leq A_{1} s\left(Q_{j}\right)$, then

$$
\sum_{j} \lambda_{j} \operatorname{Cap}\left(t_{j} Q_{j}^{\circ} \backslash X\right) \leq m A_{2} \operatorname{Cap}\left(t Q^{\circ} \backslash X\right) .
$$

Proof. (1) By the definition of capacity, it suffices to establish the estimate

$$
(s(Q))^{-1}\left\|\sum_{j} \lambda_{j} s\left(Q_{j}\right) h_{j}(x)\right\|_{\mathrm{L}_{\infty}} \leq A_{3},
$$

where $\left|h_{j}(x)\right| \leq 1, \operatorname{Spt}\left(\Delta h_{j}\right) \subset\left(t_{j} Q_{j}^{\circ} \backslash X\right)$, and $c_{0}\left(h_{j}\right)=(1 / 2) \operatorname{Cap}\left(t_{j} Q_{j}^{\circ} \backslash X\right)$. Since

$$
\left|h_{j}(x)\right| \leq A_{4} \min \left(1, \frac{s\left(Q_{j}\right)}{\left|x-a_{j}\right|}\right)
$$

( $a_{j}$ is the center of $Q_{j}$ ), we see that the left-hand side of (2.5) does not exceed the quantity

$$
A_{5}(s(Q))^{-1}\left\|\sum_{j} \lambda_{j} \int_{Q_{j}} \frac{d \sigma_{j}(z)}{|z-x|}\right\|_{\mathrm{L}_{\infty}},
$$


where $\sigma_{j}$ is the measure of total mass $\left(s\left(Q_{j}\right)\right)^{2}$ and uniformly distributed on the square cut from $Q_{j}$ by the plane passing through $a_{j}$ and parallel to $x_{3}=0$. By (2.2), the last quantity is dominated by

$$
A_{6}(s(Q))^{-1}\left\|\int_{\widetilde{Q}} \frac{d \sigma(y)}{|y-x|}\right\|_{\mathrm{L}_{\infty}} \leq A_{3}
$$

where the measure $\sigma$ is uniformly distributed on $\widetilde{Q}, y \in \widetilde{Q}$.

We have proved (2.5) and, with it, (2.3).

(2) We argue as above. In the notation of (2.5), it suffices to prove that

$$
\left\|\sum_{j} \lambda_{j} h_{j}(x)\right\|_{\mathrm{L}_{\infty}} \leq m A_{3}
$$

We have

$$
\left\|\sum_{j} \lambda_{j} h_{j}(x)\right\|_{\mathrm{L}_{\infty}} \leq A_{7}\left\|\sum_{j} \lambda_{j} \int_{Q_{j}} \frac{d \delta_{j}(z)}{|z-x|}\right\|_{\mathrm{L}_{\infty}},
$$

where the measure $\delta_{j}$ of total mass $Q_{j}$ is uniformly distributed on $\left(Q_{j}\right)$. The position of the projections of the cubes $Q_{j}$ and condition (2.2) imply that the right-hand side is dominated by $A_{8} \int_{s\left(Q_{j}\right)}^{s(Q)} d x / x$. Since $s(Q) / s\left(Q_{j}\right)=2^{m}$, estimate (2.4) follows. The lemma is proved.

Corollary to Lemma 2.2. Let $Q$ belong to $\mathbf{C o v e r}_{0}$, let $\left\{D_{j}\right\}$ be the collection of cubes that belong to Cover and are included in $Q$, and let $D \subset Q$ be an arbitrary dyadic cube that includes at least one $D_{j}$ (in particular, $D=Q$ fits). Then

$$
\sum_{\left\{j \mid D_{j} \subset D\right\}} \operatorname{Cap}\left((5 / 4) D_{j}^{\circ} \backslash X\right) s\left(D_{j}\right) \leq A \operatorname{Cap}\left((5 / 4) D^{\circ} \backslash X\right) s(D) .
$$

Proof. We remind the reader that if $D_{j} \neq Q$, then $D_{j}$ is a Whitney cube for $F$ included in $Q$. Denote by $\widetilde{Q}$ and $\widetilde{D}_{j}$ the projections of $Q$ and $D_{j}$ to the plane $x_{3}=0$. Let $\partial \widetilde{Q}$ be the boundary of $\widetilde{Q}$. Since $F$ is the frame of $\Gamma$, we have $\operatorname{dist}\left(\widetilde{D}_{j}, \partial \widetilde{Q}\right) \leq A_{1} s\left(D_{j}\right)$; since $\Gamma$ is Lipschitzian, by property (i) of Whitney's cubes we conclude that for every $x \in \widetilde{Q}$ the sum of $\chi\left(\widetilde{D}_{j}\right)(x)$ over all cubes $D_{j}$ of the same size is at most $A_{2}\left(A_{1}\right.$ and $A_{2}$ are universal constants). From (2.4) it follows that the left-hand side of (2.6) does not exceed the quantity $A_{3} \operatorname{Cap}\left((5 / 4) D^{\circ} \backslash X\right) s(D) \sum_{m=1}^{\infty} \frac{m}{2^{m}}$. This proves the corollary.

Clearly, since condition (3) in Lemma 2.1 is fulfilled for $\mathbf{C o v e r}_{0}$, the corollary to Lemma 2.2 shows that a similar condition holds true for Cover, with a greater $A_{2}$.

To finish the proof of Lemma 2.1, it suffices to remove certain coordinated pairs from $\mathbf{P a i r}_{0}$ in such a way that the resulting set Pair satisfy condition (4) of Lemma 2.1, and condition (2) of Lemma 2.1 remain true for Cover and Pair with a constant $k_{6}=k_{6}\left(k_{0}\right)$ which is controlled though is somewhat greater then the corresponding constant for Pair $_{0}$. To do this, we proceed as follows.

In Pair $_{0}$, we order the coordinated pairs in accordance with (nonstrict) increase of the cube's edge lengths. Let $\left(Q_{1}^{(1)}, Q_{2}^{(1)}\right)$ be a coordinated pair of cubes of the largest size. Fixing this pair, we eliminate all coordinated pairs (except $\left(Q_{1}^{(1)}, Q_{2}^{(1)}\right)$ itself) in which at least one cube intersects $2 Q_{1}^{(1)} \cup 2 Q_{2}^{(1)}$. After that we do much the same, fixing consecutively pairs $\left(Q_{1}^{(n)}, Q_{2}^{(n)}\right)(n=2,3, \ldots)$ among the remaining cubes and eliminating the pairs in which at least one cube intersects $\left(Q_{1}^{(n)} \cup Q_{2}^{(n)}\right)$. Having done all that, we denote by $C_{\text {cover }}$ the sum of $\beta(Q) s(Q)$ over all pairs that have not been eliminated (the set itself of these pairs is denoted by Pair). 
Condition (2) of Lemma 2.1 is fulfilled. Indeed, by (0.2), (2.3), and property (1) for Cover $_{0}$, at each step the sum $\beta(Q) s(Q)$ over all cubes eliminated at this step does not exceed the quantity $A k_{6} \min \left(\beta\left(Q_{1}^{(n)}\right), \beta\left(Q_{2}^{(n)}\right)\right) s\left(Q_{1}^{(n)}\right)$ for the pair $\left(Q_{1}^{(n)}, Q_{2}^{(n)}\right)$ fixed at the step in question.

Condition (4) of Lemma 2.1 is fulfilled by construction; condition (3) holds true for Pair because it holds true for Pair $\mathbf{P}_{0}$. This proves Lemma 2.1.

We return to the proof of Lemma 1.8. This statement will be reduced to the technical Lemma 2.3 to be proved in $\S 3$. But first we observe the following.

Let $\left\{\varphi_{j}\right\}=\left\{\varphi_{j}\right\}$ (Cover) be the partition of unity constructed in accordance with Lemma 1.5. and let $\Phi_{j}$ be the functions in (1.22) equating the coefficients $c_{0}$ for the localizations $g_{j}=E *\left(\varphi_{j} \Delta g\right), r_{j}=g_{j}-\Phi_{j}$.

Clearly, by (1.22), (1.30), and condition (3) in Lemma 2.1 for $x \notin 3 \mathbf{Q}_{0}$, we have

$$
\max \left(\sum_{\text {Cover }}\left|r_{j}(x)\right|, \sum_{\mathbf{H}(\text { Pair })}\left|\left(h_{2,1}\right)_{j}(x)\right|\right) \leq A
$$

(the first sum is over all cubes $Q_{j}$ in Cover, the second is over all functions $h_{2,1}$ in $\mathbf{H}($ Pair $))$.

But if $x \in 3 \mathbf{Q}_{0}$, then (2.7) may fail if the cover contains too many cubes. This is the main difficulty in the proof of Lemma 1.8 .

For the proof of Lemma 1.8, it is important that the function $\partial E / \partial x_{m}$ are "good Calderón-Zygmund kernels" of singular integral operators, namely, the kernels of Riesz transformations. Therefore (see, e.g., [18, Part 3, Lemma 2.6]), we have the following estimate in $\mathrm{L}_{2}=\mathrm{L}_{2}(d \sigma)$ :

$$
\left\|\partial E / \partial x_{m} * \psi\right\|_{\mathrm{L}_{2}} \leq A\|\psi\|_{\mathrm{L}_{2}} .
$$

Here $\sigma$ is the surface measure on $\Gamma, \psi \in \mathrm{L}_{2}(\Gamma, d \sigma)$, and the convolution is understood as a principal value singular integral.

Lemma 1.8 is a consequence of the next statement to be proved in $\S 3$.

Lemma 2.3. Let $\mathbf{D}=3 \mathbf{Q}_{0}$, and let $\nu \in \mathrm{L}_{1}\left(\mathbb{R}^{3}\right)$ be an arbitrary nonnegative function such that $\operatorname{Spt} \nu \subset \mathbf{D}$. Then there exists a cover $\operatorname{Cover}(\nu)$ and a set $\mathbf{P a i r}(\nu)$ of coordinated pairs such that the following is true.

(a1) Cover $(\nu)$ is obtained from Cover by replacing some cubes with their dyadic supercubes; every cube in $\operatorname{Cover}(\nu)$ is included in $\mathbf{Q}_{0}$; the set $\operatorname{Pair}(\nu)$ is obtained from Pair by removing some coordinated pairs.

(a2) The sets $\mathbf{C o v e r}(\nu), \operatorname{Pair}(\nu)$, and $\mathbf{H}(\mathbf{P a i r}(\nu))$ (the last is the corresponding set of functions $h_{2,1}$ constructed in accordance with (1.26)), and the quantity $C_{\text {cover }}(\nu)$ equal to the sum of $\beta(Q) s(Q)$ over all cubes belonging to some pair in $\operatorname{Pair}(\nu)$ satisfy conditions (1)-(4) of Lemma 2.1 with the same surface $\Gamma$, the relevant constants being at most $A_{0}$ times as much as before (moreover, $C_{\text {cover }}(\nu) \leq C_{\text {cover }}$ ).

(a3) We have

$$
\int_{\mathbf{D}}\left(\left|\sum_{\mathbf{C o v e r}(\nu)} r_{j}(x)\right|+\left|\sum_{\mathbf{H}(\operatorname{Pair}(\nu))}\left(h_{2,1}\right)_{j}(x)\right|\right) \nu(x) d m_{x} \leq k_{7}\|\nu\|_{\mathrm{L}_{1}},
$$

where the functions $r_{j}=r_{j, \nu}$ (taken from (1.22) ) are constructed for the partition of unity $\left\{\varphi_{j}\right\}(\operatorname{Cover}(\nu))$ (see Lemma 1.5) that corresponds to the new $\operatorname{Cover}(\nu)$; furthermore, $k_{7}$ depends only on $k_{0}$ in (0.2) and on $k_{2}$ in Lemma 1.5 .

The deduction of Lemma 1.8 from Lemma 2.3 is done much as Theorem 2 in [8, §2]. We recall the arguments. 
(A) For every function $\nu$, in accordance with Lemma 2.3, we construct the cover $\operatorname{Cover}(\nu)$ and the collection $\mathbf{H}(\operatorname{Pair}(\nu))$ of functions, obtaining the sequences of functions $\left\{g_{j}\right\}(\nu),\left\{\Phi_{j}\right\}(\nu)$, and $\left\{r_{j}\right\}(\nu)$ as in (1.22); by condition (a1) of Lemma 2.3, the set of all such sequences for various $\nu$ is finite. For fixed $\nu$, we consider the following functions: $G_{3, \nu}^{1}=\sum_{j} \Phi_{j}$ (here $\Phi_{j}=g_{j}-r_{j}$ as in (1.22), $g-\sum_{j} \Phi_{j}=\sum_{j} r_{j}$ ), $G_{3, \nu}^{2}=\sum_{\mathbf{H}(\operatorname{Pair}(\nu))}\left(h_{2,1}\right)_{j}$.

(B) We apply the lemma below to the functions

$$
\left|\sum_{\mathbf{C o v e r}(\nu)} r_{j}\right|+\left|\sum_{\mathbf{H}(\mathbf{P a i r}(\nu))}\left(h_{2,1}\right)_{j}\right| .
$$

Lemma 2.4. Let $\psi_{1}, \psi_{2}, \ldots, \psi_{p}$ be a finite collection of nonnegative functions on $\mathbf{D}$, and let $b>0$ be a constant.

If for every $\nu$ as in Lemma 2.3 there exists $k=k(\nu)$ such that $\int_{\mathbf{D}} \psi_{k}(x) \nu(x) d m_{x} \leq$ $b\|\nu\|_{\mathrm{L}_{1}}$, then for some convex combination $\psi=\sum_{k=1}^{p} \lambda_{k} \psi_{k}\left(\right.$ with $\lambda_{k} \geq 0$ and $\left.\sum_{k=1}^{p} \lambda_{k}=1\right)$ we have $\max _{x \in \mathbf{D}} \psi(x) \leq b$.

Proof. Let $C(\mathbf{D})$ be the set of real-valued functions $h$ continuous on $\mathbf{D}$, with the uniform norm $\|h\|=\max _{x \in \mathbf{D}}|h(x)|$. By the Riesz theorem, the dual of $C(\mathbf{D})$ is the space of real Borel measures (charges) $\mu$ supported on $\mathbf{D}(\|\mu\|$ is the total variation of $\mu)$.

Denote by $W_{1}$ the closed ball $\{h:\|h\| \leq b\}$ in $C(\mathbf{D})$, and by $W_{2}$ the convex hull of $\psi_{1}, \ldots, \psi_{p}$. Arguing by contradiction, suppose that $W_{1}$ and $W_{2}$ do not intersect. By the separation theorem (see, e.g., [19, Theorem 3.4)]), there exists a measure $\mu$ such that

$$
\sup _{h \in W_{1}}\left(\int h(x) d \mu_{x}\right)=b\|\mu\|<\int \psi(x) d \mu_{x}
$$

for every $\psi \in W_{2}$. In particular,

$$
\min _{k} \int \psi_{k}(x) d \mu_{x}>b\|\mu\|
$$

since the functions $\psi_{k}$ are nonnegative, we can replace $\mu$ by the nonnegative measure $\mu_{+}\left(\mu=\mu_{+}+\mu_{-}\right)$in this inequality. Since there are only finitely many $\psi_{k}$ 's, a similar inequality is true for some nonnegative absolutely continuous measure on $\mathbf{D}$ (a regularization of $\mu_{+}$), which contradicts the assumption.

Now, Lemma 2.4 and estimate (2.9) show that there exist $G_{3}^{1}$ and $\widetilde{G}_{3}^{2}$ (appropriate convex combinations of $G_{3, \nu}^{1}$ and $G_{3, \nu}^{2}$ with the same coefficients) with the property that $\max \left(\left\|G_{3}^{1}\right\|_{\mathrm{L}_{\infty}(\mathbf{D})},\left\|\widetilde{G}_{3}^{2}\right\|_{\mathrm{L}_{\infty}(\mathbf{D})}\right) \leq k_{7}$. By the maximum of the modulus principle, the same estimate is valid on $\mathbb{R}^{3}$.

(C) By condition (2) in Lemma 2.1 Remark 2.1, condition (a2) of Lemma 2.3, and estimate (1.24), for every function $\nu$ as in Lemma 2.3 we have $\sum_{j=1}^{3}\left|c_{j}^{1}\right|\left(g-G_{3, \nu}^{1}\right) \leq$ $k_{8} c_{3}^{1}\left(G_{3, \nu}^{2}\right)$. Representing $g$ as the convex combination $g=\sum_{k=1}^{p} \lambda_{k} g$ with the same coefficients as in (B), we see that $\sum_{j=1}^{3}\left|c_{j}^{1}\right|\left(g-G_{3}^{1}\right) \leq k_{8} c_{3}^{1}\left(\widetilde{G}_{3}^{2}\right)$; we take $G_{3}^{2}=k_{8} \widetilde{G}_{3}^{2}$.

Thus, conditions (2) and (3) of Lemma 1.8 are fulfilled for $G_{3}^{1}$ and $G_{3}^{2}$. Condition (4) of that lemma is fulfilled by (1.29); condition (1) is obvious.

This completes the deduction of Lemma 1.8 from Lemma 2.3. Thus, to complete the proof of Lemma 1.8, it remains to prove Lemma 2.3 itself.

\section{§3. Proof of Lemma 2.3. The end of the proof of Theorem 1}

Proof of Lemma 2.3. Since condition (a3) of Lemma 2.3 is linear with respect to $\nu$, there is no loss of generality in assuming that

$$
\|\nu\|_{\mathrm{L}_{1}}=\Lambda C_{\text {cover }}<1,
$$


where $C_{\text {cover }}$ is taken from condition (2) of Lemma 2.1 the universal (small) constant $\Lambda$, $0<\Lambda \leq 1$, will be determined in accordance with estimate (3.3) in Remark 3.2.

To prepare the modification described in condition (a1) of Lemma 2.3, we perform an additional construction involving $\nu$. First, we resort to the dyadic Calderón-Zygmund decomposition.

The cube $\mathbf{D}$ is the union of 27 congruent dyadic cubes $D_{j}$ with $s\left(D_{j}\right)=1$. We have $\int_{D_{j}} \nu(x) d m_{x}<\left(s\left(D_{j}\right)\right)^{2}=1$, but $s(Q)<1$ for every cube $Q$ in Cover (Cover contains more than one cube).

We split every $D_{j}$ into 8 congruent dyadic cubes; let $D$ be any of the resulting cubes. If the size of $D$ is smaller than or equal to the minimal size of the cubes in Cover, we fix $D$ and call it "good"; otherwise, we call it "bad" if $\int_{D} \nu(x) d m_{x} \geq(s(D))^{2}$, but if $\int_{D} \nu(x) d m_{x}<(s(D))^{2}$, we again split $D$ into 8 congruent dyadic cubes. For each of the resulting cubes we repeat the same procedure. Clearly, the process will terminate after finitely many steps. This construction leads to the following lemma.

Lemma 3.1. The cube $\mathbf{D}$ splits in a finite family of disjoint dyadic cubes (among which only "good" cubes $D^{g}$ and "bad" cubes $D^{b}$ may occur) with the following properties:

(b1) $s(D) \geq \min _{j} s\left(Q_{j}\right)$, where the $Q_{j}$ are cubes belonging to Cover;

(b2) $\left(s\left(D^{b}\right)\right)^{2} \leq \int_{D^{b}} \nu(x) d m_{x}<8\left(s\left(D^{b}\right)\right)^{2}$;

(b3) $\int_{D^{\prime}} \nu(x) d m_{x}<8\left(s\left(D^{\prime}\right)\right)^{2}$ if $D^{\prime}$ is an arbitrary cube including at least one cube of the splitting;

(b4) if a dyadic cube $D^{\prime}$ includes at least one "bad" cube, then $\sum_{D^{b} \subset D^{\prime}}\left(s\left(D^{b}\right)\right)^{2} \leq$ $\int_{D^{\prime}} \nu(x) d m_{x}<8\left(s\left(D^{\prime}\right)\right)^{2}$ (in particular, taking $D^{\prime}=\mathbf{Q}_{0}$, we obtain $\sum_{D \in\left\{D^{b}\right\}}(s(D))^{2} \leq$ $\left.\|\nu\|_{\mathrm{L}_{1}}\right)$.

Proof. Properties (b1) and (b2) are obvious. Should (b3) fail, some dyadic cube containing $D^{\prime}$ would have been fixed at an earlier stage as "bad" (by construction). Property (b4) follows from (b2) and (b3):

$$
\sum_{D^{b} \subset D^{\prime}}\left(s\left(D^{b}\right)\right)^{2} \leq \sum_{D^{b} \subset D^{\prime}} \int_{D^{b}} \nu(x) d m_{x} \leq \int_{D^{\prime}} \nu(x) d m_{x}<8\left(s\left(D^{\prime}\right)\right)^{2} .
$$

This proves the lemma.

Remark 3.1. We explain the role of the dyadic decomposition. It is easily seen that, in general, the estimate $\left\|\sum_{\text {Cover }} r_{j}(x)\right\|_{\mathrm{L}_{\infty}} \leq A$ may fail under the assumptions of Lemma 2.1. So, if $\int_{Q_{j}} \nu(x) d m_{x} \gg\left(s\left(Q_{j}\right)\right)^{2}$ for some $Q_{j} \in$ Cover, estimate (2.9) may also fail. But Lemma 3.1 ensures a discrete analog of the Carleson condition for cubes of the dyadic decomposition (condition (b3) of Lemma 3.1). Subsequently, we enlarge some of $Q_{j} \in$ Cover to ensure the property that, for every "bad" cube $D^{b}$, the cube $(3 / 2) D^{b}$ does not include "too small" cubes of the cover (for the same purpose, we eliminate some pairs from Pair and the corresponding functions $h_{2,1}$ ).

We continue the proof of Lemma 2.3. We generalize the construction used in [20, §2.3] in order to build a certain family halo $\left(D^{b}, \Gamma\right)$ of dyadic cubes for every "bad" cube $D^{b}$.

Denote an arbitrary "bad" cube $D^{b}$ with edge length $s$ by $D$. Let $\widetilde{D}$ be the projection of $D$ to the plane $x_{3}=0$ (so, $\widetilde{D}$ is a square). In this plane, we construct a family halo $(\widetilde{D})$ of dyadic squares as follows.

We put $\widetilde{D}^{(0)}=\widetilde{D}$. Let $\widetilde{D}^{(1)}$ be the collection consisting of $\widetilde{D}$ and all squares with side length $s / 2$ that are not included in $\widetilde{D}$ but touch $\widetilde{D}$. Similarly, for $m>1$ the collection $\widetilde{D}^{(m)}$ contains $\widetilde{D}^{(m-1)}$ and all squares with side length $s / 2^{m}$ that are not included in any square of $\widetilde{D}^{(m-1)}$ but touch some of them. Then halo $(\widetilde{D})$ is the family of all squares $\widetilde{Q}$ in 
$\bigcup_{m=0}^{\infty} \widetilde{D}^{(m)}$ with $s(\widetilde{Q})>\min _{\widetilde{D}} s\left(Q_{j}\right)$, where the minimum is taken over Cover. Clearly, halo $(\widetilde{D})$ contains at least $\widetilde{D}$. Let halo $1(D)$ be the (infinite) family of all cubes whose orthogonal projection to the plane $x_{3}=0$ is a square in halo $(\widetilde{D})$ (in other words, each square in halo $(\widetilde{D})$ is replaced with the corresponding column of cubes).

We remind the reader that, by condition (1) of Lemma 2.1. for every cube $Q$ in Cover we have $\operatorname{dist}(Q, \Gamma) \leq A_{1} s(Q)$. We define the set halo $\left(D^{b}, \Gamma\right)$ in the following way.

If $D^{b}$ does not include any cube of Cover, we take halo $\left(D^{b}, \Gamma\right)=D^{b}$; otherwise, we define halo $\left(D^{b}, \Gamma\right)$ to be the set of all cubes in halo $\left(D^{b}\right)$ that include some cube of Cover.

The following lemma says that halo $\left(D^{b}, \Gamma\right)$ contains "not too many" cubes.

Lemma 3.2. Any cube $D \in \operatorname{halo}\left(D^{b}, \Gamma\right)$ satisfies the following conditions.

(c1) If $D$ includes some cube of Cover, we have $\operatorname{dist}(D, \Gamma) \leq A_{1} s(D)\left(A_{1}\right.$ is taken from Lemma 2.1).

(c2) $D$ is included in $A D^{b}$.

(c3) We have

$$
\sum_{\left\{D \mid D \in \operatorname{halo}\left(D^{b}, \Gamma\right)\right\}}(s(D))^{2} \leq A\left(s\left(D^{b}\right)\right)^{2} .
$$

Proof. Condition (c1) follows from the fact that if $\operatorname{dist}(Q, \Gamma) \leq A_{1} s(Q)$ for some cube $Q$, then the same is true for an arbitrary cube including $Q$.

If $D=D^{b}$, (c2) is obvious. If $D \neq D^{b}$, by (c1) and the definition of halo $\left(D^{b}, \Gamma\right)$ we obtain the inequalities $\operatorname{dist}(D, \Gamma) \leq A_{1} s(D)$ and $\operatorname{dist}\left(D^{b}, \Gamma\right) \leq A_{1} s\left(D^{b}\right)$. Taking into account the position of the projections of $D$ and $D^{b}$ to the plane $x_{3}=0$, the inequality $s(D)<s\left(D^{b}\right)$, and the fact that $\Gamma$ is a Lipshitz surface (see condition (1) in Lemma 2.1), we obtain (c2).

Condition (c3) follows from (c1) and the fact that $\Gamma$ is a Lipschitz surface (the number of cubes in halo $\left(D^{b}, \Gamma\right)$ that are placed in one and the same column is bounded from above by a universal constant) and from the estimate

$$
\sum_{\left\{\widetilde{Q} \mid \widetilde{Q} \in \operatorname{halo}\left(\widetilde{D^{b}}\right)\right\}}(s(\widetilde{Q}))^{2} \leq 9\left(s\left(D^{b}\right)\right)^{2} .
$$

This completes the proof of Lemma 3.2 .

By (3.2), property (b4), and the inequality $\operatorname{Cap}((5 / 4) D) \leq 2 s(D)$, we obtain the following estimate $\left(A_{2}>0\right.$ is a universal constant):

$$
\begin{aligned}
\sum_{\left\{D^{b}\right\}\left\{D \mid D \in \operatorname{halo}\left(D^{b}, \Gamma\right)\right\}} \beta(D) s(D) & \leq 2 \sum_{\left\{D^{b}\right\}} \sum_{\left\{D \mid D \in \operatorname{halo}\left(D^{b}, \Gamma\right)\right\}}(s(D))^{2} \\
& \leq A \sum_{D \in\left\{D^{b}\right\}}(s(D))^{2} \leq A_{2}\|\nu\|_{\mathrm{L}_{1}}=\Lambda A_{2} C_{\text {cover }} .
\end{aligned}
$$

Consider the collection of all coordinated pairs in Pair that possess the following property to be called Pair - halo $\left(D^{b}, \Gamma\right)$ :

at least one of the cubes in the pair is a proper part of some cube in halo $\left(D^{b}, \Gamma\right)$.

Construction. Under the assumptions of Lemma 2.1 we make the following changes.

1. We exclude from Pair all coordinated pairs having the property $\mathbf{P a i r}-\operatorname{halo}\left(D^{b}, \Gamma\right)$; the set of remaining pairs will be denoted by $\operatorname{Pair}(\nu)$. Next, $C_{\text {cover }}(\nu)$ is the sum of $\beta(Q) s(Q)$ over all cubes belonging to pairs in $\mathbf{P a i r}(\nu)$ and $\mathbf{H}(\mathbf{P a i r})(\nu)$ is the set of the corresponding functions $h_{2,1}$.

2. We modify Cover in the following way. We add to Cover all cubes in halo $\left(D^{b}, \Gamma\right)$ for all $D^{b}$; in the resulting family, we retain only the cubes maximal with respect to 
inclusion and drop all other cubes. The outcome is the cover $\operatorname{Cover}(\nu)$ required for Lemma 2.3 .

Now, we show that, for appropriate $\Lambda$, the conditions of Lemma 2.3 are satisfied.

Condition (a1) readily follows from the construction. Recall that condition (a2) of Lemma 2.3 means that conditions (1)-(4) of Lemma 2.1 are retained.

By construction, the cubes of Cover either are preserved as they are or extended (by inclusion) to cubes of halo $\left(D^{b}, \Gamma\right)$; at the same time, the coordinated pairs in Pair that consist of cubes included in cubes of halo $\left(D^{b}, \Gamma\right)$ are dropped. Therefore, the relationship between $\Gamma$, the cubes in the cover, and those in coordinated pairs, as indicated in condition (1) of Lemma 2.1, is retained.

Condition (2) of Lemma 2.1 is preserved by (3.3) and condition (3) of Lemma 2.1 Indeed, (3.3) shows that the sum $\beta(Q) s(Q) \omega_{g}(s(Q))$ over all cubes in $\operatorname{Cover}(\nu)$ is at most $A$ times the same sum over Cover (because $\Lambda \leq 1$ ).

Consider an arbitrary cube $D$ in halo $\left(D^{b}, \Gamma\right)$. By condition (3) of Lemma 2.1 and the definition of coordinated pairs (recall that the $m_{3}$-indices (see (1.7)) for two cubes in a pair may differ at most by 3 and the $m_{1}$ - and $m_{2}$-indices may differ at most by 1 ), we see that the elimination of all cubes in the coordinated pairs in which at least one element is a proper part of $D$ reduces $C_{\text {cover }}$ at most by $A_{0}(s(D))^{2}$. By (3.3), when we treat this way all cubes in halo $\left(D^{b}, \Gamma\right)$, the quantity $C_{\text {cover }}$ is reduced at most by $A_{0} A_{1} \Lambda C_{\text {cover }}$.

Remark 3.2. $\Lambda$ will be determined by the condition $A_{0} A_{1} \Lambda=1 / 2$ (for given $A_{0}$ and $A_{1}$ in (3.3)). Then $1 / 2 C_{\text {cover }} \leq C_{\text {cover }}(\nu) \leq C_{\text {cover }}$.

Thus, we have proved that condition (2) of Lemma 2.1 is preserved (with slightly different constants).

Condition (3) of Lemma 2.1 is preserved because it is fulfilled for the cubes added to Cover, by the estimate $\operatorname{Cap}((5 / 4) Q) \leq 2 s(Q)$, conditions $(\mathrm{c} 2)$ and (c3) of Lemma 3.2. and condition (b4) of Lemma 3.1] for the cubes left without changes in Cover, this condition is fulfilled automatically.

Condition (4) of Lemma 2.1 is preserved by construction. Indeed, let $Q \in \operatorname{halo}_{1}(D)$. If there exist a cube $Q_{1} \in$ halo $_{1}(D)$ that intersects $(3 / 2) Q$, than the ratio $s(Q) / s\left(Q_{1}\right)$ may only take the values 2,1 or $1 / 2$.

The main task in the proof of Lemma 2.3 is to verify condition (a3). By Remark 3.2, it suffices to show that

$$
\int_{\mathbf{D}}\left(\left|\sum_{\mathbf{C o v e r}(\nu)} r_{j}(x)\right|+\left|\sum_{\mathbf{H}(\mathbf{P a i r})(\nu)}\left(h_{2,1}\right)_{j}(x)\right|\right) \nu(x) d m_{x} \leq k_{8} C_{\text {cover }},
$$

where $\nu$ satisfies (3.1).

The next remark is needed to simplify the notation. It explains the meaning of condition (4) of Lemma 2.1 if the capacity $\beta_{j}$ is small compared to $s_{j}$, then the estimate available for $\left|r_{j}(x)\right|$ is "much worse" for $x \in Q_{j}$ than for $x \notin(3 / 2) Q_{j}$.

Remark 3.3. The cover from Lemma 2.3 still satisfies condition (4) of Lemma 2.1, Therefore, when proving (3.4), we can replace the functions $r_{j}$ (see (1.22) ) by the differences $r_{j}-\chi\left((3 / 2) Q_{j}\right) r_{j}$ (thus, we may assume that $r_{j}(x)=0$ for $\left.x \in(3 / 2) Q_{j}\right)$. Similarly, each function $h_{2,1}$ can be replaced with $h_{2,1}-\chi\left((3 / 2) Q_{1} \cup(3 / 2) Q_{2}\right) h_{2,1}$. Not to complicate the notation, we retain the old symbols for the modified $r_{j}$ and $h_{2,1}$.

We introduce a function $\widetilde{\nu}$ by the formula

$$
\widetilde{\nu}(x)=\sum_{D \in\left(\left\{D^{g}\right\} \cup\left\{D^{b}\right\}\right)} \chi(D)(x)(s(D))^{-3} \int_{D} \nu(x) d m_{x},
$$


where $D$ runs through all cubes $\left(D^{g}\right.$ and $\left.D^{b}\right)$ in Lemma 3.1. Clearly,

$$
\int_{D}(\nu(x)-\widetilde{\nu}(x)) d m_{x}=0
$$

in particular, $\|\nu\|_{\mathrm{L}_{1}}=\|\widetilde{\nu}\|_{\mathrm{L}_{1}}$. The function $\widetilde{\nu}$ is better than $\nu$ : condition (b3) of Lemma 3.1 implies that $\widetilde{\nu}$ satisfies the Carleson condition

$$
\int_{Q} \widetilde{\nu}(x) d m_{x}<8(s(Q))^{2}
$$

for an arbitrary dyadic cube $Q$ (and not merely for the cubes that include some $D^{g}$ or $D^{b}$ from Lemma 3.1).

Clearly, (3.4) reduces to the following two estimates (which we will verify):

$$
\begin{aligned}
& \int_{\mathbf{D}}\left(\left|\sum_{\mathbf{C o v e r}(\nu)} r_{j}(x)\right|+\left|\sum_{\mathbf{H}(\mathbf{P a i r})(\nu)}\left(h_{2,1}\right)_{j}(x)\right|\right) \widetilde{\nu}(x) d m_{x} \leq k_{9} C_{\text {cover }} \\
& \left|\int_{\mathbf{D}}\left(\left|\sum_{\operatorname{Cover}(\nu)} r_{j}(x)\right|+\left|\sum_{\mathbf{H}(\text { Pair })(\nu)}\left(h_{2,1}\right)_{j}(x)\right|\right)(\nu(x)-\widetilde{\nu}(x)) d m_{x}\right| \leq k_{9} C_{\text {cover }} .
\end{aligned}
$$

We outline the idea of the proof of these two inequalities. Estimate (3.8) is, in essence, a consequence of (2.8), by (1.23), (1.24), (1.30), and (3.7). To prove it, it would have been even unnecessary to modify the cover Cover in Lemma 2.1, Cover has been replaced with $\operatorname{Cover}(\nu)$ in order to ensure (3.9), in the proof of which condition (3.6) will be used in an essential way.

Proof of (3.8). We establish the inequality

$$
\int_{\mathbf{D}}\left|\sum_{\operatorname{Cover}(\nu)} r_{j}(x)\right| \widetilde{\nu}(x) d m_{x} \leq k_{9} C_{\text {cover }} .
$$

The summand corresponding to $\left|\sum_{\mathbf{H}(\mathbf{P a i r})(\nu)}\left(h_{2,1}\right)_{j}(x)\right|$ is estimated similarly, and we shall indicate only the changes in the argument.

Consider an arbitrary cube $Q_{j}$ in $\operatorname{Cover}(\nu)$ and the corresponding function $r_{j}$ in (1.22). Let $\widetilde{Q_{j}}$ be the projection of $Q_{j}$ to $\Gamma$ parallel to the $x_{3}$-axis (similarly, $A \widetilde{Q_{j}}$ is the projection of $A Q_{j}$ for $A>0$ ); let $\widetilde{r_{j}}$ be defined by

$$
\widetilde{r_{j}}=\sum_{m=1}^{3}\left(\frac{\partial E}{\partial x_{m}} * \psi_{m, j}\right),
$$

where $\psi_{m, j}=\frac{c_{m}^{1}\left(r_{j}\right) \chi\left(\widetilde{Q_{j}}\right)}{\sigma\left(\widetilde{Q_{j}}\right)}$ and the convolution is understood as a principal value singular integral $\left(\sigma(\cdot)\right.$ is the area). Clearly, $\int_{\Gamma} \psi_{m, j}(x) d \sigma_{x}=c_{m}^{1}\left(r_{j}\right)$. From (1.24) it follows that

$$
\left|\psi_{m, j}(x)\right| \leq A k_{2} \beta_{j} \omega_{g}\left(s_{j}\right)\left(s_{j}\right)^{-1} \chi\left(\widetilde{Q_{j}}\right)(x) .
$$

By (1.23), (3.11), and the fact that $\operatorname{dist}(Q, \Gamma) \leq A_{1} s(Q)$, for $x \notin U_{j}$ (where $U_{j}=$ $\left.\left\{x \mid \operatorname{dist}\left(x, \widetilde{Q_{j}}\right) \leq s_{j}\right\}\right)$ we have

$$
\left|r_{j}(x)-\widetilde{r_{j}}(x)\right| \leq A_{1} k_{2} \omega_{g}\left(s_{j}\right) \frac{\left(s_{j}\right)^{2} \beta_{j}}{\left|x-\widetilde{a}_{j}\right|^{3}} ;
$$

here $\widetilde{a}_{j}$ is the projection of the center of $Q_{j}$ to $\Gamma$ (we remind the reader (see Remark 3.3) that the functions $r_{j}$ have been modified to satisfy, in particular, $r_{j}(x)=0$ for $x \notin$ $\left.(3 / 2) Q_{j}\right)$. 
Recall that if $\widetilde{\nu}$ satisfies (3.7) and $\psi \in \mathrm{L}_{2}(\Gamma, d \sigma)$, then, by (2.8) and, e.g., Lemma 2.5 in [18, Part 3], we have

$$
\int_{\mathbb{R}^{3}}\left|\frac{\partial E}{\partial x_{m}} * \psi(x)\right|^{2} \widetilde{\nu}(x) d m_{x} \leq A_{2} \int_{\Gamma}\left|\frac{\partial E}{\partial x_{m}} * \psi(x)\right|^{2} d \sigma_{x} \leq A_{3} \int_{\Gamma}|\psi(x)|^{2} d \sigma_{x}
$$

In particular, by the Cauchy inequality, estimate (2.8) applied to the convolution (3.11), condition (3.7), and inequality (3.12), we obtain

$$
\begin{aligned}
\int_{U_{j}}\left|\widetilde{r_{j}}(x)\right| \widetilde{\nu}(x) d m_{x} & \leq A_{4}\left(\int_{U_{j}} \widetilde{\nu}(x) d m_{x}\right)^{1 / 2}\left(\int_{U_{j}}\left|\widetilde{r_{j}}(x)\right|^{2} \widetilde{\nu}(x) d m_{x}\right)^{1 / 2} \\
& \leq A_{5} s_{j} \sum_{m=1}^{3}\left(\int_{\widetilde{Q_{j}}}\left|\psi_{m, j}(x)\right|^{2} d \sigma_{x}\right)^{1 / 2} \leq A_{6} k_{2} \beta_{j} \omega_{g}\left(s_{j}\right) s_{j} .
\end{aligned}
$$

(A similar estimate for $r_{j}$ is obvious by (1.22) and Remark 3.3.)

By (3.7), formulas (3.13) and (3.15) imply

$$
\int_{\mathbf{D}}\left|r_{j}(x)-\widetilde{r_{j}}(x)\right| \widetilde{\nu}(x) d m_{x} \leq A_{7} k_{2} \omega_{g}\left(s_{j}\right)\left(s_{j}\right)^{2} \beta_{j} \sum_{k=1}^{\infty} \frac{\left(2^{k} s_{j}\right)^{2}}{\left(2^{k} s_{j}\right)^{3}}=A_{7} k_{2} \beta_{j} \omega_{g}\left(s_{j}\right) s_{j} .
$$

Summing in (3.16) over the indices $j$ corresponding to all cubes in $\operatorname{Cover}(\nu)$, by condition (2) of Lemma 2.1 we obtain

$$
\int_{\mathbf{D}} \sum_{j}\left|r_{j}(x)-\widetilde{r_{j}}(x)\right| \widetilde{\nu}(x) d m_{x} \leq A_{8} k_{2} \sum_{j} \beta_{j} \omega_{g}\left(s_{j}\right) s_{j} \leq A_{9} k_{2} k_{6} C_{\text {cover }} .
$$

Consequently, in order to prove (3.10), it suffices to show that

$$
\int_{\mathbf{D}}\left|\sum_{j} \widetilde{r}_{j}(x)\right| \widetilde{\nu}(x) d m_{x} \leq k_{10} C_{\text {cover }}
$$

(summation is over all cubes in $\operatorname{Cover}(\nu)$ ).

Much as in (3.14) and (3.15), from (3.12) we deduce the estimate

$$
\begin{aligned}
& \int_{\mathbf{D}}\left|\sum_{j} \widetilde{r}_{j}(x)\right| \widetilde{\nu}(x) d m_{x} \\
& \quad \leq A_{1} k_{2}\left(\int_{\mathbf{D}} \widetilde{\nu}(x) d m_{x}\right)^{1 / 2}\left(\int_{\Gamma}\left(\sum_{j} \frac{\beta_{j} \omega_{g}\left(s_{j}\right)}{s_{j}} \chi\left(\widetilde{Q_{j}}\right)(x)\right)^{2} d \sigma_{x}\right)^{1 / 2}
\end{aligned}
$$

Thus, by (3.1), to prove (3.10) it suffices to verify the inequality

$$
\int_{\Gamma}\left(\sum_{j} \frac{\beta_{j} \omega_{g}\left(s_{j}\right)}{s_{j}} \chi\left(\widetilde{Q_{j}}\right)(x)\right)^{2} d \sigma_{x} \leq k_{11} C_{\text {cover }}
$$


We observe that if the interiors of the projections $\widetilde{Q_{j}}$ and $\widetilde{Q_{k}}$ intersect, then one of these projections is included in the other. As a result, we arrive at (3.18):

$$
\begin{aligned}
& \int_{\Gamma}\left(\sum_{j} \frac{\beta_{j} \omega_{g}\left(s_{j}\right)}{s_{j}} \chi\left(\widetilde{Q_{j}}\right)(x)\right)^{2} d \sigma_{x} \\
& \quad \leq 2 \sum_{j} \frac{\beta_{j} \omega_{g}\left(s_{j}\right)}{s_{j}} \sum_{\left\{k \mid \widetilde{Q_{k}} \subset \widetilde{Q_{j}}\right\}} \int_{\widetilde{Q_{j}}} \frac{\beta_{k} \omega_{g}\left(s_{k}\right)}{s_{k}} \chi\left(\widetilde{Q_{k}}\right)(x) d \sigma_{x} \\
& \quad \leq A_{3} \sum_{j} \frac{\beta_{j} \omega_{g}\left(s_{j}\right)}{s_{j}} \sum_{\left\{k \mid Q_{k} \subset A Q_{j}\right\}} \beta_{k} \omega_{g}\left(s_{k}\right) s_{k} \\
& \quad \leq A_{4} \sum_{j} \frac{\beta_{j} \omega_{g}\left(s_{j}\right)}{s_{j}}\left(s_{j}\right)^{2} \leq k_{11} C_{\text {cover }} .
\end{aligned}
$$

Comments: since $\operatorname{dist}(Q, \Gamma) \leq A_{1} s(Q)$ for all cubes in the cover (condition (1) of Lemma 2.1), the inclusion $\widetilde{Q_{k}} \subset \widetilde{Q_{j}}$ implies $Q_{k} \subset A Q_{j}$; in the next to the last inequality we have employed condition (3) of Lemma 2.1 and condition (a2) of Lemma 2.3 .

The estimate

$$
\int_{\mathbf{D}}\left|\sum_{\mathbf{H}(\text { Pair })(\nu)}\left(h_{2,1}\right)_{j}(x)\right| \widetilde{\nu}(x) d m_{x} \leq k_{9} C_{\text {cover }}
$$

is proved much as (3.10), because the functions $h_{2,1}$ are designed much as the $r_{j}$. Specifically, as in (3.11), for every $h_{2,1}$ we consider the function

$$
\widetilde{h_{2,1}}=\sum_{m=1}^{3}\left(\frac{\partial E}{\partial x_{m}} * \psi_{m}\right) \text {, }
$$

where $\psi_{m}=\frac{c_{m}^{1}\left(h_{2,1}\right) \chi(\widetilde{Q})}{\sigma(\widetilde{Q})}$, and $\widetilde{Q}$ is the projection of some cube in the coordinated pair to $\Gamma$. By (1.29), we have the inequality $\left|\psi_{m}(x)\right| \leq A \beta(Q)(s(Q))^{-1} \chi(\widetilde{Q})(x)$ similar to 3.12 .

The estimate

$$
\int_{\mathbf{D}}\left|h_{2,1}(x)-\widetilde{h_{2,1}}(x)\right| \widetilde{\nu}(x) d m_{x} \leq A_{1} \beta(Q) s(Q)
$$

is proved much as (3.16). When we sum over all functions $h_{2,1}$, we must take into account the definition of $C_{\text {cover }}$ in condition (3) of Lemma 2.1.

The estimate

$$
\int_{\mathbf{D}}\left|\sum_{\mathbf{H}(\text { Pair })(\nu)}\left(\widetilde{h_{2,1}}\right)_{j}(x)\right| \widetilde{\nu}(x) d m_{x} \leq k_{10} C_{\text {cover }}
$$

is verified as (3.17). This completes the proof of (3.8).

Proof of (3.9). We shall use the following properties of the cubes in Cover $(\nu)$ and the cubes belonging to coordinated pairs in $\operatorname{Pair}(\nu)$. This properties stem from the construction (passage to $\operatorname{Cover}(\nu)$ and $\operatorname{Pair}(\nu)$ from Cover and Pair); in essence, the construction was aimed at these properties.

Let $Q$ be a cube in $\operatorname{Cover}(\nu)$ or some cube belonging to a coordinated pair in $\mathbf{P a i r}(\nu)$. Let $D$ belong to halo $\left(D^{b}, \Gamma\right)$ for some $D^{b}$ (in particular, it may happen that $D=D^{b}$ ). Then

(1) $Q$ is not a proper part of $D$;

(2) if $(3 / 2) D$ intersects $Q$, then $s(D) \leq 2 s(Q)$. 
We prove the estimate

$$
\left|\int_{\mathbf{D}}\right| \sum_{\mathbf{H}(\mathbf{P a i r})(\nu)}\left(h_{2,1}\right)_{j}(x)\left|(\nu(x)-\widetilde{\nu}(x)) d m_{x}\right| \leq k_{9} C_{\text {cover }}
$$

The inequality

$$
\left|\int_{\mathbf{D}}\right| \sum_{\operatorname{Cover}(\nu)} r_{j}(x)\left|(\nu(x)-\widetilde{\nu}(x)) d m_{x}\right| \leq k_{9} C_{\text {cover }}
$$

is proved similarly, but it is simpler because, unlike (3.20), individual cubes of the cover are involved rather then pairs $\left(Q_{1}^{\prime}, Q_{2}^{\prime}\right) \in \operatorname{Pair}(\nu)$. So, in the case of the second inequality, we only pay attention to changes in the arguments.

Consider arbitrary cubes $D_{p}$ in $\left\{D^{g}\right\} \cup\left\{D^{b}\right\}$ (their centers will be denoted by $a_{p}^{\prime}$ ). By (3.6) and elementary properties of the modulus, (3.20) reduces to the following estimate (summation is over all functions $h_{2,1}$ in $\mathbf{H}(\mathbf{P a i r})(\nu)$ and all cubes $D_{p}$ ):

$$
\left.\sum_{j} \sum_{p} \int_{D_{p}}\left|\left(h_{2,1}\right)_{j}(x)-\left(h_{2,1}\right)_{j}\left(a_{p}^{\prime}\right)\right| \mid \nu(x)\right)-\widetilde{\nu}(x) \mid d m_{x} \leq k_{9} C_{\text {cover }} .
$$

Let $Q$ be a cube of the pair $\left(Q_{1}^{\prime}, Q_{2}^{\prime}\right) \in \operatorname{Pair}(\nu)$. Then the two cubes in $\left(Q_{1}^{\prime}, Q_{2}^{\prime}\right)$ are included in $A_{1} Q$ with $A_{1}=7$; if $Q$ is a cube in $\operatorname{Cover}(\nu)$ corresponding to $r_{j}$, then in the similar estimate

$$
\left.\sum_{j} \sum_{p} \int_{D_{p}}\left|r_{j}(x)-r_{j}\left(a_{p}^{\prime}\right)\right| \mid \nu(x)\right)-\widetilde{\nu}(x) \mid d m_{x} \leq k_{9} C_{\text {cover }}
$$

we take naturally $A_{1}=1$.

Let $D$ be an arbitrary cube $D_{p}$ (i.e., $D^{g}$ or $D^{b}$ in Lemma 3.1). We say that $Q$ and $D$ are "close" if $(5 / 4) D$ intersects $2 A_{1} Q$; otherwise we say they are "remote".

First, we consider the case of "remote" cubes $Q$ and $D$. Since the distances between points of $A_{1} Q$ and (5/4)D are all "roughly the same", by (1.27), (1.28), and (1.30) we obtain

$$
\left.\int_{D}\left|\left(h_{2,1}\right)(x)-\left(h_{2,1}\right)\left(a^{\prime}\right)\right| \mid \nu(x)\right)-\widetilde{\nu}(x) \mid d m_{x} \leq A_{2} s(D) \frac{\beta(Q) s(Q)}{\left|a-a^{\prime}\right|^{3}} \int_{D} \nu(x) d m_{x}
$$

where $a$ and $a^{\prime}$ are the centers of $Q$ and $D$ (when considering the functions $r_{j}$, we replace the quantity $\beta(Q) s(Q)$ on the right in (3.21) by $\left.k_{2} \omega\left(s_{j}\right) \beta\left(Q_{j}\right) s\left(Q_{j}\right)\right)$.

We fix $D$ and sum over all $Q$ "remote" from $D$; it follows that the sum is dominated by

$$
A_{3} \int_{D} \nu(x) d m_{x} s(D) \int_{\{y|| y \mid \geq s(D)\}} \frac{d \sigma_{y}}{\left|y-a^{\prime}\right|^{3}} \leq A_{4} \int_{D} \nu(x) d m_{x},
$$

where we have used the estimate $\sigma\left(B\left(a^{\prime}, r\right)\right) \leq A_{5} r^{2}$; see condition (3) of Lemma 2.1 and condition (a2) of Lemma 2.3 (in the case of the functions $r_{j}$, the arguments are similar).

It remains to sum the integrals $\int_{D} \nu(x) d m_{x}$ over all cubes $D$ from Lemma 3.1 and to use condition (3.1). This finishes the proof of (3.20) in the case of "remote" cubes.

Consider "close" cubes $Q$ and $D$. Two cases are possible.

1. The cube $(3 / 2) D$ intersects $Q$.

2. The cube $(5 / 4) D$ intersects $2 A_{1} Q$, but $(3 / 2) D$ does not intersect $Q$.

Whereas simply $s(D) \leq 2 s(Q)$ in the first case, in the second $D$ also cannot be "too large" compared to $Q$ (this is clear geometrically). Thus, for "close" cubes we have $D \subset A_{6} Q$. Consequently, $D$ is included in the union of at most 8 dyadic cubes with edge length of at most $2 A_{6} s(Q)$. Thus, by (b3) in Lemma 3.1 and estimate (3.7), for every cube $Q$ the sum of the integrals $\int_{D}|\nu(x)-\widetilde{\nu}(x)| d m_{x}$ over all $D$ "close" to $Q$ is at most $A_{7}(s(Q))^{2}$. 
By Remark 3.3, we may assume that $h_{2,1}(x)=0$ for $x \in\left((3 / 2) Q_{1} \cup(3 / 2) Q_{2}\right)$ and also $r_{j}(x)=0$ for $x \in(3 / 2) Q_{j}$. Now, $\left|h_{2,1}(x)\right| \leq A_{8} \beta(Q)(s(Q))^{-1}$ by (1.27) and (1.30). Similarly, by (1.22), $\left.\left|r_{j}(x)\right| \leq A_{8} k_{2} \omega_{g}\left(s\left(Q_{j}\right)\right) \beta\left(Q_{j}\right)\left(s\left(Q_{j}\right)\right)^{-1}\right)$ for the function $r_{j}$ corresponding to the cube $Q_{j}$ in $\operatorname{Cover}(\nu)$. By (b3) of Lemma 3.1 and condition (3.7), it follows that

$$
\left.\sum_{j} \sum_{\{D \mid D \text { is "close" to } Q\}} \int_{D} \mid\left(h_{2,1}\right)_{j}(x)(\nu(x))-\widetilde{\nu}(x)\right) \mid d m_{x} \leq A_{9} \sum_{j} \beta\left(Q_{j}\right) s\left(Q_{j}\right)=A_{10} C_{\text {cover }}
$$

(summation is over all $\left(h_{2,1}\right)_{j}$ in $\mathbf{H}$ (Pair) $(\nu)$ ); a similar estimate holds true for $r_{j}$.

We have proved (3.9) and, with it, Lemma 2.3. Thus, we have deduced Lemma 1.8 from Lemma 2.1.

Lemma 1.8 is proved much as Lemma 1.8. The difference is that, when constructing $G_{3}^{1}$ and $G_{3}^{2}$, in place of dyadic cubes we use parallelepipeds of the form

$$
\begin{aligned}
\mathcal{Q}_{p}^{m_{1}, m_{2}, m_{3}}=\left[m_{1} 2^{-p},\left(m_{1}+1\right) 2^{-p}\right] & \times\left[m_{2} 2^{-p},\left(m_{2}+1\right) 2^{-p}\right] \\
& \times\left[16 m_{3} 2^{-p}, 16\left(m_{3}+1\right) 2^{-p}\right] .
\end{aligned}
$$

We define coordinated pairs of parallelepipeds (3.22) precisely as we did this for cubes: $\left(\max \left(\left|m_{1}-m_{1}^{\prime}\right|,\left|m_{2}-m_{2}^{\prime}\right|\right)=1\right.$ and $\left.2 \leq\left|m_{3}-m_{3}^{\prime}\right| \leq 3\right)$, and introduce functions $h_{2,1}$ as in (1.26). Then $c_{3}^{1}\left(h_{2,1}\right) \geq 2 \max _{j \neq 3}\left|c_{j}^{1}\right|\left(h_{2,1}\right)$ (in place of $1 / 8$ for dyadic cubes). Thus, for the function $G_{3}^{2}$ (a linear combination of $h_{2,1}$ with positive coefficients), condition (4) of Lemma 1.8 will be fulfilled.

To construct the other pairs $\left(G_{m}^{1}, G_{m}^{2}\right), m=1,2$, we use parallelepipeds "dilated" with coefficients 16 along other coordinate axes.

Repeating thrice the arguments proving Lemma 1.8 (each time relative to a particular coordinate axis) for parallelepipeds, we arrive at Lemma 1.8. This proves Theorem 1 (recall that it was reduced to Lemma 1.8 in $\S 1$ ).

\section{$\S 4$. Proof of Theorem 2}

We show that the claim of Theorem 1 remains true if, in condition (0.1), we replace the class of all functions $\varphi$ by the family of functions $\varphi_{\delta}^{a}$ of the form $\varphi_{\delta}^{a}(x)=\delta^{-3} \varphi_{1}^{0}\left(\frac{x-a}{\delta}\right)$, where

$$
\varphi_{1}^{0}(x)=\frac{15}{8 \pi} \chi(B(0,1))\left(1-x_{1}^{2}-x_{2}^{2}-x_{3}^{2}\right)
$$

(clearly, $\varphi_{1}^{0}(x) \geq 0, \varphi_{1}^{0} \in C_{0}^{1}(B(0,1))$ and $\int \varphi_{1}^{0}(x) d m_{x}=1$ ).

The Green formula for harmonic functions (see, e.g., formula (29)) in [21, §6, item 5]) shows that

$$
\left\langle\Delta f \mid \varphi_{\delta}^{a}\right\rangle=15 \delta^{-2}\left(\frac{1}{\sigma(\partial B)} \int_{\partial B} f(x) d \sigma_{x}-\frac{1}{m(B)} \int_{B} f(x) d m_{x}\right)
$$

where $(B=B(a, \delta))$. As in [11], we construct certain auxiliary functions. For $m \in \mathbb{N}$, we put

$$
\varphi_{m, \delta}^{0}=\underbrace{\varphi_{\delta}^{0} * \varphi_{\delta}^{0} * \cdots * \varphi_{\delta}^{0}}_{m} .
$$

Clearly, $\varphi_{m, \delta}^{0}(x) \geq 0$, Spt $\varphi_{m, \delta}^{0} \subset B(0, m \delta)$, and $\int \varphi_{m, \delta}^{0}(x) d m_{x}=1$. Let $\left\{\varphi_{j}\right\}$ be the standard $\delta$-partition of unity on $\mathbb{R}^{3}$ (see, e.g., [3, Chapter 2, §1] or [14, Lemma 3.1]). This means the following. Let $j=\left(j_{1}, j_{2}, j_{3}\right) \in \mathbb{Z}^{3}$, and let $a_{j}=\left(j_{1} \delta, j_{2} \delta, j_{3} \delta\right)$; then the closures of the balls $B\left(a_{j}, \delta \sqrt{3} / 2\right)$ cover $\mathbb{R}^{3}$. Consequently, there exist nonnegative functions $\varphi_{j} \in C_{0}^{\infty}\left(B_{j}\right)$ (where $B_{j}=B\left(a_{j}, \delta\right)$ ) with $\left\|\nabla^{n} \varphi_{j}\right\|_{\mathrm{L}_{\infty}} \leq A(n) \delta^{-n}$ and $\sum_{j} \varphi_{j} \equiv 1$ on $\mathbb{R}^{3}$. 
With the help of functions $\varphi_{m, \delta}^{0}$, we construct the new partition of unity $\left\{\psi_{m, j}\right\}=$ $\left\{\varphi_{m, \delta}^{0} * \varphi_{j}\right\}$, where $\psi_{m, j} \in C_{0}^{\infty}\left((m+1) B_{j}\right)$; the derivatives of the $\psi_{m, j}$ obey the same estimates as in the case of the $\varphi_{j}$.

Theorem 2 is a consequence of the following statement.

Theorem 3. Let the initial function $f$ and the functions $\varphi_{\delta}^{a}$ satisfy the estimates

$$
\left|\left\langle\Delta f \mid \varphi_{\delta}^{a}\right\rangle\right| \leq k_{1} \omega_{f}(\delta) \delta^{-3} \operatorname{Cap}(B(a, k \delta) \backslash X),
$$

where $k$ and $k_{1}$ may only depend on $f$ and $X$.

Then for an arbitrary ball $B$ of radius $r$ and an arbitrary function $\varphi \in C_{0}^{3}(B)$ we have

$$
|\langle\Delta f \mid \varphi\rangle| \leq A k_{1} k^{3} \omega_{f}(r)\left\|\nabla^{3} \varphi\right\|_{\mathrm{L}_{\infty}} r^{3} \operatorname{Cap}((k+3) B \backslash X) .
$$

Indeed, putting the right-hand side of (4.1) to the left-hand side of (4.3), we obtain (0.3). Recall (see Lemmas 1.1 and 1.5) formulas (1.22) and (1.30) ) that, in fact, in the proof of Theorem 1. estimate (0.1) was applied only to functions $\varphi$ whose derivatives obey the estimates $\left\|\nabla^{n} \varphi\right\|_{\mathrm{L}_{\infty}} \leq A(n) \delta^{-n}(n=0,1,2, \ldots)$, where $\delta$ is the minimal radius of a ball the closure of which includes Spt $\varphi$; moreover, $r \leq A_{1} k \delta$. Clearly, for such functions we can replace (0.1) by (4.4) in Theorem 1, and Theorem 2 follows.

To prove Theorem 31, we need three lemmas. The first two, taken jointly, are similar to Lemma 2.5 in [11]; the third is similar to Lemma 2 in [3. Chapter 2, §6] or Lemma 2.4 in [22, Chapter 2] about the additivity of the capacity for special partitions of sets.

Lemma 4.1. Suppose (4.3) is true. Let $\Psi \in C\left(\mathbb{R}^{3}\right)$ satisfy Spt $\Psi \subset(B(a, m \delta))$ for some $m \in \mathbb{N}$. Then

$$
\left|\left\langle\Delta f \mid \varphi_{\delta}^{0} * \Psi\right\rangle\right| \leq k_{1} \omega_{f}(\delta) \delta^{-3} \operatorname{Cap}(B(a,(k+m) \delta) \backslash X)\|\Psi\|_{\mathrm{L}_{1}} .
$$

Proof. For every $f \in C^{2}$ we have

$$
\begin{aligned}
\left\langle\Delta f \mid \varphi_{\delta}^{0} * \Psi\right\rangle & =\iint \Delta_{x} f(x+y) \varphi_{\delta}^{0}(x) \Psi(y) d m_{x} d m_{y} \\
& =\int \Psi(y) d m_{y} \int \Delta f(t) \varphi_{\delta}^{0}(t-y) d m_{t} \\
& =\int \Psi(y) d m_{y} \int \Delta f(t) \varphi_{\delta}^{y}(t) d m_{t}=\int \Psi(y) d m_{y}\left\langle\Delta f \mid \varphi_{\delta}^{y}\right\rangle
\end{aligned}
$$

by passage to a limit, we see that the same is true for $f \in C$ (indeed, the estimates in (4.1) depend only on the uniform norm of $f)$. Since $y \in \operatorname{Spt} \Psi \subset B(a, m \delta)$, we obtain (4.3) by (4.5).

Lemma 4.1 makes it possible to construct (by convolution) new functions for which (4.4) is true. In doing this, we need certain estimates for solutions of convolution equations. In some cases, such estimates can be obtained as in [11, Lemma 2.5].

Lemma 4.2. Let $\left\{\varphi_{j}\right\}$ be the $\delta$-partition of unity considered above, let $\alpha$ be a multiindex with $|\alpha| \leq 2$, and let $\Psi_{\alpha, j}$ be the solution of the following convolution equation:

$$
\left(\varphi_{3, \delta}^{0} * \varphi_{j}\right)\left(x-a_{j}\right)^{\alpha}=\varphi_{\delta}^{0} * \Psi_{\alpha, j} .
$$

Then $\Psi_{\alpha, j} \in C_{0}^{\infty}\left(B\left(a_{j}, 3 \delta\right)\right)$ and $\left\|\Psi_{\alpha, j}\right\|_{\mathrm{L}_{\infty}} \leq A \delta^{|\alpha|}$.

Proof. A translation reduces the matter to the case where $a_{j}=0$. We assume that $|\alpha|>0$ (otherwise the claim is trivial). We denote by $\widehat{(\cdot)}$ the Fourier transform of a 
function. Also, recall that $\widehat{x_{m} g(x)}=-i \frac{\partial \widehat{g}}{\partial x_{m}}, m=1,2,3$. Taking Fourier transforms, we obtain that (in the sense of distributions)

$$
\widehat{\Psi_{\alpha, j}}=(-i)^{|\alpha|} \frac{\partial^{\alpha}\left(\widehat{\varphi_{j}}\left(\widehat{\varphi_{\delta}^{0}}\right)^{3}\right)}{\widehat{\varphi_{\delta}^{0}}} .
$$

Note that we took $m=3$ in formula (4.2) to ensure the possibility of division by $\widehat{\varphi_{\delta}^{0}}$ for $|\alpha| \leq 2$. After simple calculations and passage to inverse Fourier transforms, we represent $\Psi_{\alpha, j}$ as a finite linear combination of functions of the following type:

$$
\left(x^{\gamma_{1}} \varphi_{\delta}^{0}\right) *\left(x^{\gamma_{2}} \varphi_{\delta}^{0}\right) *\left(x^{\gamma_{3}} \varphi_{j}\right), \quad \text { where }\left|\gamma_{1}\right|+\left|\gamma_{2}\right|+\left|\gamma_{3}\right|=|\alpha| .
$$

Now, the claim follows by the definition of $\varphi_{\delta}^{0}$ and $\varphi_{j}$ and by the standard properties of convolution.

Lemmas 4.1 and 4.2 imply the following estimate for the partition of unity $\left\{\psi_{3, j}\right\}=$ $\left\{\varphi_{3, \delta}^{0} * \varphi_{j}\right\},|\alpha| \leq 2$ :

$$
\left|\left\langle\psi_{3, j} \Delta f \mid\left(x-a_{j}\right)^{\alpha}\right\rangle\right|=\left|\left\langle\Delta f \mid \varphi_{\delta}^{0} * \Psi_{\alpha, j}\right\rangle\right| \leq k_{1} \omega_{f}(\delta) \delta^{|\alpha|} \operatorname{Cap}\left(B\left(a_{j},(k+3) \delta\right) \backslash X\right)
$$

(indeed, $\left\|\Psi_{\alpha, j}\right\|_{\mathrm{L}_{1}} \leq A \delta^{3+|\alpha|}$ by Lemma 4.2).

Proof of Theorem 3, Fixing $\delta=\operatorname{Cap}((k+3) B \backslash X)$, we observe that (4.4) is meaningful only if $\delta$ is "much smaller" than $r$ (indeed, clearly, $\langle\Delta f \mid \varphi\rangle \leq A \omega_{f}(r)\left\|\nabla^{2} \varphi\right\|_{L_{\infty}} r^{3} \leq$ $\left.A_{1} \omega_{f}(r)\left\|\nabla^{3} \varphi\right\|_{\mathrm{L}_{\infty}} r^{4}\right)$.

Consider the partition of unity $\left\{\psi_{3, j}\right\}$. By the Taylor formula, we have

$$
\begin{aligned}
\langle\Delta f \mid \varphi\rangle= & \sum_{j}\left\langle\psi_{3, j} \Delta f \mid \varphi\right\rangle \\
= & \sum_{j} \varphi\left(a_{j}\right)\left\langle\Delta f \mid \psi_{3, j}\right\rangle \\
& +\sum_{j} \sum_{\{\alpha|1 \leq| \alpha \mid \leq 2\}} \frac{\partial^{\alpha} \varphi\left(a_{j}\right)}{\alpha !}\left\langle\psi_{3, j} \Delta f \mid\left(x-a_{j}\right)^{\alpha}\right\rangle+\sum_{j}\left\langle\Delta f \mid \psi_{3, j} R_{3, j}\right\rangle,
\end{aligned}
$$

where $\partial^{\alpha} R_{3, j}\left(a_{j}\right)=0$ for $|\alpha| \leq 2$ and $\partial^{\alpha} R_{3, j} \equiv \partial^{\alpha} \varphi$ for $|\alpha|=3$; consequently, for $x \in \operatorname{Spt} \psi_{3, j} \subset B\left(a_{j}, 4 \delta\right)$ and $|\alpha| \leq 2$ we have $\left|\partial^{\alpha} R_{3, j}(x)\right| \leq A \delta^{3-|\alpha|} \mid \nabla^{3} \varphi \|_{\mathrm{L}_{\infty}}$. Taking the estimates for the derivatives of $\psi_{3, j}$ into account, we obtain $\left|\Delta\left(\psi_{3, j} R_{3, j}\right)(x)\right| \leq$ $A \delta\left\|\nabla^{3} \varphi\right\|_{\mathrm{L}_{\infty}}$.

Since the number of the indices $j$ for which $(k+3) B_{j}$ intersects $B$ is at most $A(k r / \delta)^{3}$, we arrive at the estimate

$$
\left|\sum_{j}\left\langle\Delta f \mid \psi_{3, j} R_{3, j}\right\rangle\right| \leq A_{1} \omega_{f}(\delta) \delta^{3}\left\|\nabla^{3} \varphi\right\|_{\mathrm{L}_{\infty}} \delta\left(\frac{k r}{\delta}\right)^{3}=A_{1} \omega_{f}(\delta) k^{3}\left\|\nabla^{3} \varphi\right\|_{\mathrm{L}_{\infty}} r^{3} \delta ;
$$

by the choice of $\delta$, this does not exceed the right-hand side of (4.4).

To estimate the other terms on the right in (4.7), we shall need the following statement (to be verified after the proof of Theorem 3).

Lemma 4.3. Under the assumptions of Theorem 13, we have

$$
\sum_{j} \operatorname{Cap}\left(B\left(a_{j},(k+3) \delta\right) \backslash X\right) \leq A k^{3} \delta
$$

(summation is over the indices $j$ for which $(k+3) B_{j}$ intersects $\left.B\right)$. 
From this, (4.6), and the relation $\delta=\operatorname{Cap}((k+3) B \backslash X)$, we obtain the following estimates:

$$
\begin{aligned}
\left|\sum_{j} \varphi\left(a_{j}\right)\left\langle\Delta f \mid \psi_{3, j}\right\rangle\right| & \leq A_{1} k_{1} k^{3} \omega_{f}(\delta)\|\varphi\|_{\mathrm{L}_{\infty}} \delta ; \\
\left|\sum_{j} \sum_{\{\alpha|1 \leq| \alpha \mid \leq 2\}} \frac{\partial^{\alpha} \varphi\left(a_{j}\right)}{\alpha !}\left\langle\psi_{3, j} \Delta f \mid\left(x-a_{j}\right)^{\alpha}\right\rangle\right| & \leq A_{1} k_{1} k^{3} \omega_{f}(\delta)\left\|\nabla^{2} \varphi\right\|_{\mathrm{L}_{\infty}} \delta^{3} .
\end{aligned}
$$

This establishes (4.4). The proof of Theorem 3 is finished.

Proof of Lemma 4.3. We mimic the proof of Lemma 2.4 in [22, Part 2]. Let $E_{j}=$ $B\left(a_{j},(k+3) \delta\right) \backslash X$, and let $g_{j}$ be functions satisfying $\left\|g_{j}\right\|_{\mathrm{L}_{\infty}} \leq 1$, Spt $\Delta g_{j} \subset E_{j}$, $\lim _{x \rightarrow \infty} g_{j}(x)=0$, and $\left\langle\Delta g_{j} \mid 1\right\rangle \geq(1 / 2) \operatorname{Cap} E_{j}$. Then

$$
\sum_{j}\left|g_{j}(x)\right| \leq A_{1}\left(k^{3}+\sum_{\left\{j \mid x \notin 2(k+3) B_{j}\right\}} \frac{\operatorname{Cap} E_{j}}{\left|x-a_{j}\right|}\right)
$$

for arbitrary $x \in \mathbb{R}^{3}$. Applying the Hölder inequality, we obtain

$$
\begin{aligned}
\sum_{\left\{j \mid x \notin 2(k+3) B_{j}\right\}} \frac{\operatorname{Cap} E_{j}}{\left|x-a_{j}\right|} & \leq\left(\sum_{j}\left(\frac{\operatorname{Cap} E_{j}}{\delta}\right)^{4 / 3}\right)^{3 / 4}\left(\sum_{\left\{j \mid x \notin 2(k+3) B_{j}\right\}} \frac{\delta^{4}}{\left|x-a_{j}\right|^{4}}\right)^{1 / 4} \\
& \leq A_{2} k^{3}\left(\sum_{j} \frac{\operatorname{Cap} E_{j}}{\delta}\right)^{3 / 4} .
\end{aligned}
$$

Thus,

$$
\left\|\sum_{j} g_{j}\right\|_{\mathrm{L}_{\infty}} \leq A_{3} k^{3}\left(1+\left(\sum_{j} \frac{\operatorname{Cap} E_{j}}{\delta}\right)^{3 / 4}\right) .
$$

It follows from this, the properties of functions $g_{j}$, and the definition of capacity that

$$
\sum_{j} \operatorname{Cap} E_{j} \leq 2 A_{3} k^{3}\left(1+\left(\sum_{j} \frac{\operatorname{Cap} E_{j}}{\delta}\right)^{3 / 4}\right) \delta \text {. }
$$

We denote the ratio $\frac{\sum_{j} \operatorname{Cap} E_{j}}{\delta}$ by $t$; by (4.8), we have

$$
t \leq A_{4} k^{3}\left(1+t^{3 / 4}\right) \text {. }
$$

Since $3 / 4<1$, we see that $t$ "cannot be too large", and the claim of Lemma 4.3 follows. With this, the proof of Theorem 3 is finished completely, and Theorem 2 is also established.

\section{REFERENCES}

[1] M. V. Keldysh, On the solvability and the stability of the Dirichlet problem, Uspekhi Mat. Nauk vyp. 8 (1941), 171-231. (Russian)

[2] J. Deny, Systèmes totaux de fonctions harmoniques, Ann. Inst. Fourier (Grenoble) 1 (1949), 103113. MR.0037414 (12:258c)

[3] A. G. Vitushkin, Analytic capacity of sets in problems of approximation theory, Uspekhi Mat. Nauk 22 (1967), no. 6, 141-199; English transl. in Russian Math. Surveys 22 (1967), no. 6. MR.0229838 $(37: 5404)$

[4] A. G. O'Farrell, Uniform approximation by harmonic functions, Problem Book 3. Part 2, Lecture Notes in Math., vol. 1574, Springer-Verlag, 1994, Problem 12.15, p. 121.

[5] P. V. Paramonov, Harmonic approximations in the $C^{1}$-norm, Mat. Sb. 181 (1990), no. 10, 13411365; English transl., Math. USSR-Sb. 71 (1992), no. 1, 183-207. MR1085885 (92j:31006)

[6] E. M. Stein, Singular integrals and differentiability properties of functions, Princeton Math. Ser., No. 30, Princeton Univ. Press, Princeton, NJ, 1970. MR0290095 (44:7280) 
[7] R. Harvey and J. Polking, A notion of capacity which characterizes removable singularities, Trans. Amer. Math. Soc. 169 (1972), 183-195. MR0306740 (46:5862)

[8] M. Ya. Mazalov, A criterion for uniform approximability on arbitrary compact sets for solutions of elliptic equations, Mat. Sb. 199 (2008), no. 1, 15-46; English transl., Sb. Math. 199 (2008), no. 1-2, 13-44. MR2410145 (2009c:35005)

[9] _ On uniform approximations by bi-analytic functions on arbitrary compact sets in $\mathbb{C}$, Mat. Sb. 195 (2004), no. 5, 79-102; English transl., Sb. Math. 195 (2004), no. 5-6, 687-709. MR2091640 (2005g:30044)

[10] J. Verdera, The uniform approximation problem for the square of the Cauchy-Riemann operator, Problem Book 3. Part 2, Lecture Notes in Math., vol. 1574, Springer-Verlag, 1994, Problem 12.16, pp. 122-123. MR1214077(94d:30070)

[11] P. V. Paramonov, Some new criteria for the uniform approximability of functions by rational fractions, Mat. Sb. 186 (1995), no. 9, 97-112; English transl., Sb. Math. 186 (1995), no. 9, 13251340. MR 1360189 (96m:41017)

[12] J. Verdera, M. S. Mel'nikov, and P. V. Paramonov, $C^{1}$-approximation and the extension of subharmonic functions, Mat. Sb. 192 (2001), no. 4, 37-58; English transl., Sb. Math. 192 (2001), no. 3-4, 515-535. MR 1834090 (2002g:41030)

[13] L. Carleson, Selected problems on exceptional sets, Van Nostrand Math. Stud., No. 13D, Van Nostrand Co., Inc., Princeton, 1967. MR0225986 (37:1576)

[14] R. Harvey and J. Polking, Removable singularities of solutions of linear partial differential equations, Acta Math. 125 (1970), 39-56. MR0279461 (43:5183)

[15] N. N. Tarkhanov, Laurent series for solutions of elliptic systems, Nauka, Novosibirsk, 1991. (Russian) MR $1226897(94 \mathrm{e}: 35013)$

[16] P. V. Paramonov and K. Yu. Fedorovskiŭ, On uniform and $C^{1}$-approximability of functions on compact sets in $\mathbb{R}^{2}$ by solutions of second-order elliptic equations, Mat. Sb. 190 (1999), no. 2, 123-144; English transl., Sb. Math. 190 (1999), no. 1-2, 285-307. MR.1701003 (2000k:41024)

[17] N. S. Landkof, Foundations of modern potential theory, Nauka, Moscow, 1966; English transl., Grundlehren Math. Wiss., Bd. 180, Springer-Verlag, New York-Heidelberg, 1972. MR0214795 (35:5644) MR0350027 (50:2520)

[18] G. David, Wavelets and singular integrals on curves and surfaces, Lecture Notes in Math., vol. 1465, Springer-Verlag, Berlin, 1991. MR,1123480 (92k:42021)

[19] W. Rudin, Functional analysis, McGraw-Hill Book Co., New York, 1973. MR0365062 (51:1315)

[20] J. Mateu and J. Orobitg, Lipshitz approximation by harmonic functions and some applications to spectral synthesis, Indiana Univ. Math. J. 39 (1990), 703-736. MR.1078735 (92e:46052)

[21] V. S. Vladimirov, The equations of mathematical physics, 5th ed., Nauka, Moscow, 1988; English transl., Pure Appl. Math., vol. 3, Marcel Dekker, Inc., New York, 1971. MR0978200 (90m:00009) MR0268497 (42:3394)

[22] J. Verdera, Removability, capacity and approximation, Complex Potential Theory (Montreal, PQ, 1993), NATO Adv. Sci. Inst. Ser. C Math. Phys. Sci., vol. 439, Kluwer, Dordrecht, 1994, pp. 419473. MR 1332967 (96b:30086)

USSR Marshal A. M. Vasilevskil Military Academy of Air Defence, Military Forces of RF, Ul. Kotovskogo 2, Smolensk 214027, Russia

E-mail address: maksimmazalov@yandex.ru

Received MAR/15/2010

Translated by S. KISLYAKOV 